$$
=\pi
$$

NASA Contractor Report 187460

ICASE Report 90-76

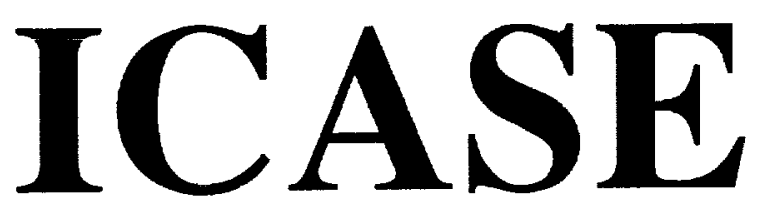

\title{
TOWARD THE LARGE-EDDY SIMULATION OF COMPRESSIBLE TURBULENT FLOWS
}

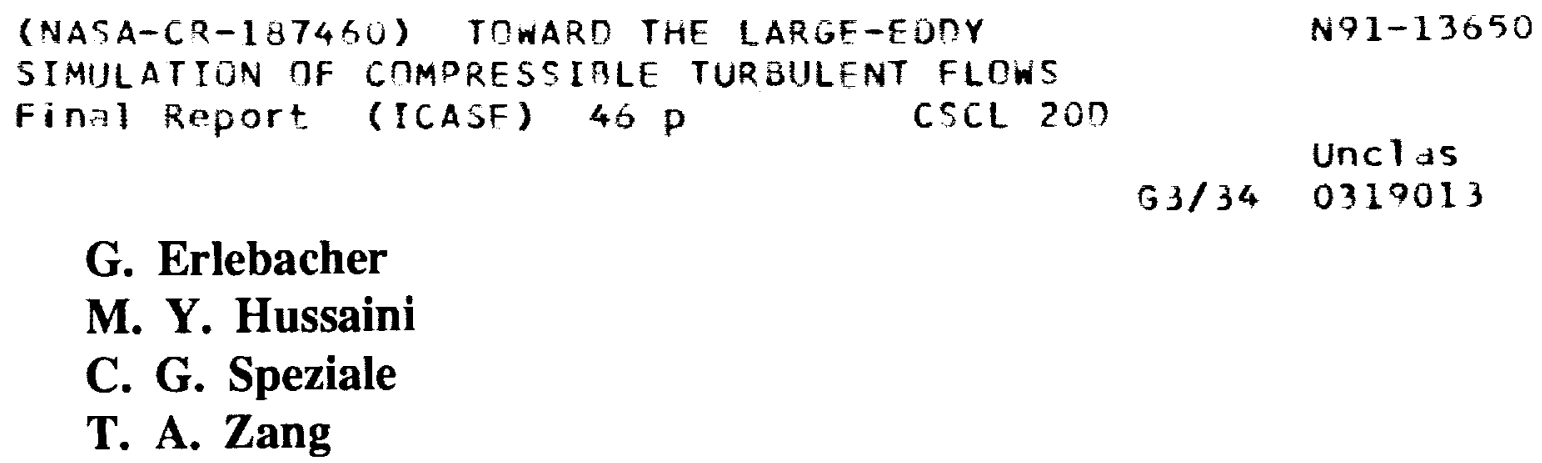

G. Erlebacher

M. Y. Hussaini

C. G. Speziale

T. A. Zang

Contract No. NAS1-18605

October 1990

Institute for Computer Applications in Science and Engineering

NASA Langley Research Center

Hampton, Virginia 23665-5225

Operated by the Universities Space Research Association

\section{N/Sก}

National Aeronautics and

Space Adminis/ration 


\title{
TOWARD THE LARGE-EDDY SIMULATION OF COMPRESSIBLE TURBULENT FLOWS ${ }^{\dagger}$
}

\author{
G. Erlebacher ${ }^{\ddagger}$ \\ ICASE, NASA Langley Research Center \\ M. Y. Hussaini ${ }^{\dagger}$ \\ ICASE, NASA Langley Research Center \\ C. G. Speziale $\mathbf{e}^{\ddagger}$ \\ ICASE, NASA Langley Research Center \\ T. A. Zang \\ NASA Langley Research Center \\ Hampton, VA 23665
}

\begin{abstract}
New subgrid-scale models for the large-eddy simulation of compressible turbulent flows are developed and tested based on the Favre-filtered equations of motion for an ideal gas. A compressible generalization of the linear combination of the Smagorinsky model and scalesimilarity model, in terms of Favre-filtered fields, is obtained for the subgrid-scale stress tensor. An analogous thermal linear combination model is also developed for the subgrid-scale heat flux vector. The two dimensionless constants associated with these subgrid-scale models are obtained by correlating with the results of direct numerical simulations of compressible isotropic turbulence performed on a $96^{3}$ grid using Fourier collocation methods. Extensive comparisons between the direct and modeled subgrid-scale fields are provided in order to validate the models. A large-eddy simulation of the decay of compressible isotropic turbulence - conducted on a coarse $32^{3}$ grid - is shown to yield results that are in excellent agreement with the fine grid direct simulation. Future applications of these compressible subgrid-scale models to the large-eddy simulation of more complex supersonic flows are discussed briefly.
\end{abstract}

\footnotetext{
†This report supersedes ICASE Report No. 87-20

tThis research was supported by the National Aeronautics and Space Administration under NASA Contract No. NAS1-18605 while the authors were in residence at the Institute for Computer Applications in Science and Engineering (ICASE), NASA Langley Research Center, Hampton, VA 23665.
} 



\section{Introduction}

The direct numerical simulation of turbulent flows at the high Reynolds numbers encountered in problems of technological importance is all but impossible as a result of the wide range of scales that are present. Consequently, the solutions to such problems must invariably be based on some form of turbulence modeling. Traditional turbulence models based on Reynolds averages have had only limited success since the large scales of the turbulence - which contain most of the energy - are highly dependent on the geometry of the flow being considered. Experience has indicated that such models usually break down when a variety of turbulent flows are considered (Lumley 1983). The small scales are more universal in character, and serve mainly as a source for dissipation. Hence, it can be argued that a better understanding of turbulent flows could be achieved if just the small scales are modeled while the large scales are calculated (Deardorff 1970). This is the fundamental idea behind large-eddy simulations.

During the past decade, considerable progress has been made in the large-eddy simulation of incompressible turbulent flows. This effort has shed new light on the physics of turbulence. The earliest work relied heavily on the use of the Reynolds averaging assumption to eliminate the Leonard and cross stresses while the Reynolds stresses were computed using the Smagorinsky model (Deardorff 1970, Leonard 1974, Reynolds 1976). More recent large-eddy simulations have been based on the direct calculation of the Leonard stresses with models provided for the cross and Reynolds subgrid-scale stresses in order to enhance the numerical accuracy (see Biringen and Reynolds 1981, Bardina Ferziger and Reynolds 1983). However, among these newer models, only the Bardina, Ferziger and Reynolds (1983) model, with a Bardina constant of 1.0, satisfies the important physical constraint of Galilean invariance (Speziale 1985). The underlying physical concepts, fundamental numerical algorithms, and comprehensive historical data behind the recent field of large-eddy simulation have been presented in articles by Schumann (1975), Voke and Collins (1983) and Rogallo and Moin (1984). More recently, work on the subgrid-scale modeling of transition to turbulence of initially laminar incompressible flows has begun (Piomelli, Zang, Speziale and Hussaini 1990). Several large-eddy simulations have been performed and initial results are promising.

Despite the intensive research effort that has been devoted to the large-eddy simulation of incompressible flows as outlined above, it appears that no large-eddy simulation of a compressible turbulent flow has yet been attempted. Of course, such work could have important technological applications in the analysis of turbulent supersonic flows, where shock waves are generated, and in turbulent flows within combustion chambers. The prerequisite for carrying out such computations is the development of suitable subgrid-scale models for compressible turbulent flows. With the exception of the recent work of Yoshizawa (1986) and Speziale et al. (1988), few, if any, studies along these lines appear to have been published. The subgrid-scale models of Yoshizawa are only suitable for slightly compressible turbulent flows since they made use of an asymptotic expansion about an incompressible state. Recently however, Dahlburg, Zang and Dahlburg (1990) have performed an extensive 
parameter study using the model developed by Speziale et al. (1988). It was shown by Speziale et al. (1988) that, for the purposes of accuracy, the Leonard and cross stresses must be accounted for. Furthermore, the modeling of the isotropic part of the Reynolds subgrid-scale stress tensor was shown to be questionable - an issue that was left for future research.

In this paper, complete subgrid-scale models are developed for the closure of the Favrefiltered Navier-Stokes and energy equations. The compressible subgrid-scale stress model that is obtained in Section 2 reduces to the linear combination model of Bardina, Ferziger and Reynolds (1983) in the incompressible limit. Likewise, the subgrid-scale heat flux model that is obtained herein consists of an analogous linear combination of scale similarity and gradient transport terms. The dimensionless constant which appears in the subgrid-scale stress model is arrived at through correlation analysis of data generated from direct numerical simulations of compressible isotropic turbulence. A more detailed comparison of computed and modeled subgrid-scale fields is presented along with the results of a large-eddy simulation of compressible isotropic turbulence.

\section{Subgrid-Scale Models for Compressible Turbulence}

The compressible turbulent flow of an ideal gas is considered. Such flows are governed by the continuity, momentum and energy equations which - neglecting body forces - are given by (cf. Batchelor 1967)

$$
\begin{gathered}
\frac{\partial \rho}{\partial t}+\frac{\partial\left(\rho v_{k}\right)}{\partial x_{k}}=0 \\
\frac{\partial\left(\rho v_{k}\right)}{\partial t}+\frac{\partial\left(\rho v_{k} v_{l}\right)}{\partial x_{l}}=-\frac{\partial p}{\partial x_{k}}+\frac{\partial \sigma_{k l}}{\partial x_{l}} \\
\frac{\partial(\rho h)}{\partial t}+\frac{\partial\left(\rho h v_{k}\right)}{\partial x_{k}}=\frac{\partial p}{\partial t}+v_{k} \frac{\partial p}{\partial x_{k}}+\frac{\partial}{\partial x_{k}}\left(\kappa \frac{\partial T}{\partial x_{k}}\right)+\Phi
\end{gathered}
$$

respectively, where $\rho$ is the mass density, $\mathbf{v}$ is the velocity vector, $p$ is the thermodynamic pressure, $\mu$ is the dynamic viscosity, $h$ is the enthalpy, $T$ is the absolute temperature, and $\kappa$ is the thermal conductivity. The viscous stress $\sigma_{k l}$ and the viscous dissipation $\Phi$ are defined by

$$
\begin{aligned}
\sigma_{k l} & =-\frac{2}{3} \mu \frac{\partial v_{j}}{\partial x_{j}} \delta_{k l}+\mu\left(\frac{\partial v_{k}}{\partial x_{l}}+\frac{\partial v_{l}}{\partial x_{k}}\right) \\
\Phi & =-\frac{2}{3} \mu\left(\frac{\partial v_{j}}{\partial x_{j}}\right)^{2}+\mu\left(\frac{\partial v_{k}}{\partial x_{l}}+\frac{\partial v_{l}}{\partial x_{k}}\right) \frac{\partial v_{k}}{\partial x_{l}}
\end{aligned}
$$

respectively. Herein, the Einstein summation convention applies to repeated indices. Equations (1)-(3) must be supplemented with the equations of state

$$
P=\rho R T, \quad h=C_{p} T
$$


for an ideal gas where $R$ is the ideal gas constant and $C_{p}$ is the specific heat at constant pressure. Likewise, the dependence of the viscosity and thermal conductivity on the temperature must be provided (i.e., relationships of the form $\mu=\mu(T)$ and $\kappa=\kappa(T)$ are needed and these depend on the gas under consideration).

Any flow variable $\mathcal{F}$ can be filtered in the following manner:

$$
\overline{\mathcal{F}}(\mathbf{x})=\int_{D} G(\mathbf{x}-\mathbf{z}, \Delta) \mathcal{F}(\mathbf{z}) d^{3} z
$$

where $G$ is a filter function, $\Delta$ is the computational mesh size, and $D$ is the domain of the fluid. The filter function $G$ is typically taken to be an infinitely differentiable function of bounded support in a bounded domain, or a Gaussian distribution in a periodic domain ${ }^{\ddagger}$. It is normalized by requiring that

$$
\int_{D} G(\mathbf{x}-\mathbf{z}, \Delta) d^{3} z=1
$$

It follows that in the limit as the computational mesh size goes to zero, (7) becomes a Dirac delta sequence, i.e.

$$
\lim _{\Delta \rightarrow 0} \int_{D} G(\mathbf{x}-\mathbf{z}, \Delta) \mathcal{F}(\mathbf{z}) d^{3} z=\int_{D} \delta(\mathbf{x}-\mathbf{z}) \mathcal{F}(\mathbf{z}) d^{3} z=\mathcal{F}(\mathbf{x})
$$

where $\delta(\mathbf{x}-\mathbf{z})$ is the Dirac delta function (Arfken 1970). The filter function has the property that the amplitude of the high-frequency spatial Fourier components of any flow variable $\mathcal{F}$ are substantially reduced. Consequently, $\overline{\mathcal{F}}$ represents the large-scale part of $\mathcal{F}$. At this point, it should be mentioned that as a result of the defining properties of $G$, it follows that

$$
\frac{\overline{\partial \mathcal{F}}}{\partial t}=\frac{\partial \overline{\mathcal{F}}}{\partial t}, \quad \frac{\overline{\partial \mathcal{F}}}{\partial x_{k}}=\frac{\partial \overline{\mathcal{F}}}{\partial x_{k}} .
$$

Piomelli, Ferziger and Moin (1987) discuss the relationship between the form of the filter function and that of the subgrid-scale turbulence model.

The turbulent fields are decomposed as follows, based on Favre filtering:

$$
\mathcal{F}=\tilde{\mathcal{F}}+\mathcal{F}^{\prime}
$$

where the Favre filter

$$
\tilde{\mathcal{F}}=\frac{\overline{\rho \mathcal{F}}}{\bar{\rho}}
$$

is defined in an analogous manner to the Favre time average which has been of use in the more traditional studies of compressible turbulent flows (Hinze 1975). However, contrary to the more traditional Favre time averaging,

$$
\tilde{\tilde{\mathcal{F}}} \neq \tilde{\mathcal{F}}
$$

\footnotetext{
${ }^{\ddagger}$ A Gaussian filter is adopted for the calculations in this study
} 
in general, and hence

$$
\overline{\mathcal{F}}^{\prime} \neq 0 .
$$

The direct filtering of the continuity equation (1) yields

$$
\frac{\partial \bar{\rho}}{\partial t}+\frac{\partial\left(\bar{\rho} \tilde{v}_{k}\right)}{\partial x_{k}}=0
$$

where we have used (10) and (12). Likewise, a direct filtering of the momentum equation yields

$$
\frac{\partial\left(\bar{\rho} \tilde{v}_{k}\right)}{\partial t}+\frac{\partial\left(\bar{\rho} \tilde{v}_{k} \tilde{v}_{l}\right)}{\partial x_{l}}=-\frac{\partial \bar{p}}{\partial x_{k}}+\frac{\partial \bar{\sigma}_{k l}}{\partial x_{l}}+\frac{\partial \tau_{k l}}{\partial x_{l}}
$$

where

$$
\bar{p}=\bar{\rho} R \tilde{T}
$$

and

$$
\tau_{k l}=-\bar{\rho}\left(\widetilde{\tilde{v}_{k} \tilde{v}_{l}}-\tilde{v}_{k} \tilde{v}_{l}+\widetilde{v_{k}^{\prime} \tilde{v}_{l}}+\widetilde{v_{l}^{\prime} \tilde{v}_{k}}+\widetilde{v_{k}^{\prime} v_{l}^{\prime}}\right)
$$

is the subgrid-scale stress tensor. The subgrid-scale stress tensor can be decomposed as follows,

$$
\boldsymbol{T}=\mathbf{L}+\mathbf{C}+\mathbf{R}
$$

where

$$
\begin{aligned}
& L_{k l}=-\bar{\rho}\left(\tilde{\tilde{v}_{k} \tilde{v}_{l}}-\tilde{v}_{k} \tilde{v}_{l}\right) \\
& C_{k l}=-\bar{\rho}\left(\bar{v}_{k}^{\prime} \bar{v}_{l}+\bar{v}_{l}^{\prime} \tilde{v}_{k}\right) \\
& R_{k l}=-\bar{\rho} \widetilde{v_{k}^{\prime} v_{l}^{\prime}}
\end{aligned}
$$

are respectively, the subgrid-scale Leonard, cross, and Reynolds stresses based on Favre filtering. From (20), it is clear that the Leonard stress can be calculated directly and does not need to be modeled. The cross stress is modeled with the scale similarity model

$$
C_{k l}=-\bar{\rho}\left(\tilde{v}_{k} \tilde{v}_{l}-\tilde{\tilde{v}}_{k} \tilde{\tilde{v}}_{l}\right)
$$

(with a coefficient of unity to ensure Galilean invariance of the overall model). This model is analogous to its incompressible counterpart, which has been reasonably successful in the large-eddy simulation of incompressible turbulent flows (Bardina, Ferziger and Reynolds 1983, Speziale 1985). The subgrid-scale Reynolds stress tensor is separated into deviatoric and isotropic parts, respectively, as follows:

$$
\mathbf{R}={ }_{D} \mathbf{R}+{ }_{I} \mathbf{R}
$$

where

$$
{ }_{D} R_{k l}=-\bar{\rho}\left(\widetilde{v_{k}^{\prime} v_{l}^{\prime}}-\frac{1}{3} \widetilde{v_{i}^{\prime} v_{i}^{\prime} \delta_{k l}}\right)
$$

and

$$
{ }_{I} R_{k l}=-\frac{1}{3} \bar{\rho} \widetilde{v_{i}^{\prime} v_{i}^{\prime}} \delta_{k l}
$$


Here, the deviatoric part of the subgrid-scale Reynolds stress tensor, ${ }_{D} \mathbf{R}$, is modeled using the compressible generalization of the Smagorinsky model that is given by

$$
{ }_{D} R_{k l}=2 C_{R} \bar{\rho} \Delta^{2} I I_{\tilde{S}}^{1 / 2}\left(\tilde{S}_{k l}-\frac{1}{3} \tilde{S}_{m m} \delta_{k l}\right)
$$

where

$$
\begin{gathered}
\tilde{S}_{k l}=\frac{1}{2}\left(\frac{\partial \tilde{v}_{k}}{\partial x_{l}}+\frac{\partial \tilde{v}_{l}}{\partial x_{k}}\right) \\
I I_{S}=\tilde{S}_{m n} \tilde{S}_{m n}
\end{gathered}
$$

(i.e., $\tilde{\mathbf{S}}$ is the Favre filtered rate of strain tensor while $I I_{\tilde{S}}$ is its second invariant) and $C_{R}$ is the compressible Smagorinsky constant. Yoshizawa - by means of a two-scale DIA method derived a model for the isotropic part of the subgrid-scale Reynolds stress tensor, $\mathbf{R}$, given by

$$
{ }_{I} R_{k l}=-\frac{2}{3} C_{I} \bar{\rho} \Delta^{2} I I_{\tilde{S}} \delta_{k l}
$$

where $C_{I}$ is a dimensionless constant. Equation (30) can, for the most part, be obtained by making a turbulence production equals dissipation equilibrium hypothesis (Yoshizawa 1986). However, this model was shown by Speziale et al. (1988) to correlate very poorly with the results of direct numerical simulations of compressible isotropic turbulence. Since ${ }_{I} \mathbf{R}$ is extremely small compared to the thermodynamic pressure, we propose to neglect it an assumption that will be justified later. Hence, the overall subgrid-scale stress model we propose takes the form

$$
\tau_{k l}=-\bar{\rho}\left(\widetilde{\tilde{v}_{k} \tilde{v}_{l}}-\tilde{\tilde{v}}_{k} \tilde{\tilde{v}}_{l}\right)+2 C_{R} \bar{\rho} \Delta^{2} I I_{\tilde{S}}^{1 / 2}\left(\tilde{S}_{k l}-\frac{1}{3} \tilde{S}_{m m} \delta_{k l}\right)
$$

In the incompressible limit, Eq. (31) reduces to the linear combination model

$$
-\tau_{k l} / \rho=\overline{\bar{v}}_{k} \bar{v}_{l}-\overline{\bar{v}}_{k} \overline{\bar{v}}_{l}-2 C_{R} \Delta^{2} I I_{\bar{S}}^{1 / 2} \bar{S}_{k l}
$$

of Bardina, Ferziger, and Reynolds (1983) where the Bardina constant is one in order to satisfy Galilean invariance (Speziale 1985). This reduction process is a consequence of Eq. (31) and

$$
\tilde{\mathbf{v}}=\overline{\mathbf{v}}, \quad \tilde{S}_{m m}=\bar{S}_{m m}=0
$$

when $\rho$ becomes constant.

A direct filtering of the energy equation yields the filtered form

$$
\begin{aligned}
\frac{\partial\left(C_{p} \bar{\rho} \tilde{T}\right)}{\partial t}+\frac{\partial\left(C_{p} \bar{\rho} \tilde{v}_{k} \tilde{T}\right)}{\partial x_{k}}= & \frac{\partial \bar{p}}{\partial t}+\overline{v_{k} \frac{\partial p}{\partial x_{k}}}+\bar{\Phi} \\
& +\frac{\partial}{\partial x_{k}}\left(\kappa \frac{\partial T}{\partial x_{k}}\right)-\frac{\partial Q_{k}}{\partial x_{k}}
\end{aligned}
$$

where

$$
Q_{k}=C_{p} \bar{\rho}\left(\widetilde{v_{k} \tilde{T}}-\tilde{v}_{k} \tilde{T}+\widetilde{v_{k}^{\prime} \tilde{T}}+\widetilde{v_{k} \bar{T}^{\prime}}+\widetilde{v_{k}^{\prime} T^{\prime}}\right)
$$


is the subgrid-scale heat flux. The subgrid-scale heat flux can be decomposed in the same fashion as the subgrid-scale stresses. This leads to

$$
\mathbf{Q}=\mathbf{Q}^{(L)}+\mathbf{Q}^{(C)}+\mathbf{Q}^{(R)}
$$

where

$$
\begin{aligned}
& \mathrm{Q}^{(L)}=C_{p} \bar{\rho}\left(\widetilde{\tilde{v}_{k} \tilde{T}}-\tilde{v} \tilde{T}\right) \\
& \mathrm{Q}^{(C)}=C_{p} \bar{\rho}\left(\tilde{v_{k}^{\prime} \tilde{T}}+\tilde{v_{k} \bar{T}^{\prime}}\right) \\
& \mathrm{Q}^{(R)}=C_{p} \bar{\rho} \overline{v_{k}^{\prime} \tilde{T}^{\prime}}
\end{aligned}
$$

are the Leonard, cross, and Reynolds heat fluxes. Analogous to the modeling of the cross stress, the cross heat flux is modeled using the scale similarity format

$$
Q_{k}^{(C)}=C_{p} \bar{\rho}\left(\tilde{v}_{k} \tilde{T}-\tilde{\tilde{v}}_{k} \tilde{\tilde{T}}\right)
$$

The Reynolds heat flux is modeled with the usual gradient transport format as follows (cf. Eidson 1985):

$$
\widetilde{v_{k}^{\prime} T^{\prime}}=-\frac{C_{R}}{P r_{T}} \Delta^{2} I I_{\tilde{S}}^{1 / 2} \frac{\partial \tilde{T}}{\partial x_{k}}
$$

where $\operatorname{Pr}_{T}$ is the turbulent Prandtl number. Of course, the Leonard heat flux can be calculated directly. Hence, the overall model for the subgrid-scale heat flux we propose is as follows:

$$
Q_{k}=C_{p} \bar{\rho}\left[\left(\widetilde{\tilde{v}_{k} \tilde{T}}-\tilde{\hat{v}_{k}} \tilde{\tilde{T}}\right)-\frac{C_{R}}{P r_{T}} \Delta^{2} I I_{\tilde{s}}^{1 / 2} \frac{\partial \tilde{T}}{\partial x_{k}}\right],
$$

and is obtained by combining equations (37), (40), and (41).

At this point, some comments need to be made concerning the viscous terms on the right-hand side of (16) and the pressure gradient-velocity and viscous dissipation terms which appear on the right-hand side of (34). The pressure gradient-velocity correlation can be written in the alternative form

$$
\begin{aligned}
\overline{v_{k} \frac{\partial p}{\partial x_{k}}} & =\frac{\partial\left(\overline{\bar{p} v_{k}}\right)}{\partial x_{k}}-\overline{p \frac{\partial v_{k}}{\partial x_{k}}} \\
& =\frac{\partial}{\partial x_{k}}\left(\overline{\rho R T v_{k}}\right)-p \frac{\partial v_{k}}{\partial x_{k}} \\
& =\frac{\partial}{\partial x_{k}}\left(\bar{\rho} R \tilde{v}_{k} \tilde{T}\right)+\frac{\partial}{\partial x_{k}}\left(\frac{R}{C_{p}} Q_{k}\right)-\overline{\frac{\partial v_{k}}{\partial x_{k}}}
\end{aligned}
$$

where only the pressure dilatation term

$$
\begin{aligned}
\overline{p \frac{\partial v_{k}}{\partial x_{k}}} & =\bar{\rho} R\left(T \frac{{\widetilde{\partial v_{k}}}_{\partial x_{k}}}{}\right. \\
& =\bar{\rho} R \tilde{T} \frac{{\widetilde{\partial \tilde{v}_{k}}}_{\partial x_{k}}}{\bar{\rho}} \bar{\rho} R T^{\prime} \frac{{\widetilde{\partial \tilde{v}_{k}}}_{\partial x_{k}}}{\bar{\rho} \rho} R \tilde{T} \frac{\partial v_{k}^{\prime}}{\partial x_{k}}+\bar{\rho} R T^{\prime} \frac{{\widetilde{\partial v_{k}}}_{\partial x_{k}}}{}
\end{aligned}
$$


is not yet closed. The temperature dilatation correlation $\left(T^{\prime} \frac{\widetilde{\partial v_{k}^{\prime}}}{\partial x_{k}}\right)$ is extremely difficult to model and not much success has been achieved in dealing with it in the context of Reynolds stress models. However, within the framework of subgrid-scale modeling, this term and its corresponding cross correlation have physical interpretations. They represent the contribution of the dilatation of the small scales to the internal energy variation of the fluid - an effect which is expected to be small. Hence, for this initial study, we neglect such terms. Furthermore, since the mean temperature is constant and the temperature fluctuations are small $(<10 \%)$, the viscosity and thermal conductivity are held constant. For similar reasons, we also neglect the small scale component of the viscous dissipation.

The turbulence model proposed herein is thus complete once values for the constants $C_{R}$ and $\operatorname{Pr}_{T}$ are obtained. This will be accomplished using the results of direct numerical simulations of compressible isotropic turbulence.

\section{Numerical Method}

Our direct simulations of compressible turbulence are based on a non-dimensional form of Eqs. (1)-(3), with the time derivative in the energy equation written solely in terms of the pressure. In order to alleviate the severe stability limit imposed at very low Mach numbers by the acoustic waves, a splitting method is adopted. The first step integrates the equations

$$
\begin{gathered}
\frac{\partial \rho}{\partial t}=0 \\
\frac{\partial\left(\rho v_{k}\right)}{\partial t}+\frac{\partial\left(\rho v_{k} v_{l}\right)}{\partial x_{l}}=\frac{\partial \sigma_{k l}}{\partial x_{l}} \\
\frac{\partial p}{\partial t}+v_{k} \frac{\partial p}{\partial x_{k}}+\gamma p \frac{\partial v_{k}}{\partial x_{k}}-c_{0}^{2} \frac{\partial\left(\rho v_{k}\right)}{\partial x_{k}}=\frac{1}{\operatorname{RePr} M_{\infty}^{2}} \frac{\partial^{2} T}{\partial x_{k} \partial x_{k}}+(\gamma-1) \Phi
\end{gathered}
$$

while the second step integrates

$$
\begin{gathered}
\frac{\partial \rho}{\partial t}+\frac{\partial\left(\rho v_{k}\right)}{\partial x_{k}}=0 \\
\frac{\partial\left(\rho v_{k}\right)}{\partial t}+\frac{\partial p}{\partial x_{k}}=0 \\
\frac{\partial p}{\partial t}+c_{0}^{2} \frac{\partial\left(\rho v_{k}\right)}{\partial x_{k}}=0
\end{gathered}
$$

The constants $c_{0}$ and $M_{\infty}$ are the current root mean square (rms) value of the sound speed (c) and the reference Mach number, while $\gamma=C_{p} / C_{v}$, where $C_{v}$ is the specific heat at constant volume. These equations are non-dimensionalized in terms of a length scale $\left(L_{0}\right)$, a velocity scale $\left(U_{0}\right)$, a pressure scale $\left(P_{0}\right)$, a reference viscosity $\mu$ and a reference thermal conductivity 
$\kappa$. The Reynolds number is given by $R e=\rho_{0} U_{0} L_{0} / \mu$, the Prandtl number by $\operatorname{Pr}=C_{p} \mu / \kappa$, and the reference Mach number by $M_{\infty}=U_{0} /\left(\gamma R T_{0}\right)^{1 / 2}$. For all calculations presented in this study, $\gamma=1.4$, and $\operatorname{Pr}=0.7$. Initially, the density $\rho_{0}$ is uniform and equal to one. The computational domain is a cube, normalized to $[0,2 \pi]^{3}$. Periodic boundary conditions are imposed in all three directions.

The spatial derivatives in these equations are approximated by a Fourier collocation method (see, for example, Hussaini and Zang 1987). In each coordinate direction, $N$ grid points are used: $x_{k_{j}}=2 \pi j / N$, for $j=0,1, \ldots, N-1$. The derivative of a function $\mathcal{F}(\mathbf{x})$ with respect to $x_{k}$ is approximated by the analytic derivative of the trigonometric interpolant of $\mathcal{F}(\mathbf{x})$ in the direction $x_{k}$. Most simulations of incompressible, homogeneous turbulence have used a Fourier Galerkin method. The compressible equations, however, contain cubic rather than quadratic nonlinearities and true Galerkin methods are more expensive (compared with collocation methods) than they are for incompressible flow. The essential difference between collocation and Galerkin methods is that the former are subject to both truncation and aliasing errors, whereas the latter have only truncation errors. As discussed extensively by Canuto, et al (1987), the aliasing terms are not significant for a well-resolved flow. However, care is needed to pose a collocation method in a form which ensures numerical stability. For this reason, the second term in (46) is actually used in the equivalent form

$$
\frac{1}{2}\left[\frac{\partial\left(\rho v_{k} v_{l}\right)}{\partial x_{l}}+\rho v_{l} \frac{\partial v_{k}}{\partial x_{l}}+v_{k} \frac{\partial\left(\rho v_{l}\right)}{\partial x_{l}}\right] \text {. }
$$

As noted by Feiereisen, Reynolds and Ferziger (1981), when this form is employed together with a symmetric differencing method in space (for example Fourier collocation), then in addition to mass, and momentum, energy is conserved for the ideal compressible equations (zero viscosity and thermal conductivity) in the absence of time differencing (and splitting) errors.

The second fractional step of the splitting, given by (48)-(50), contains most of the effects of the acoustic waves. This splitting is employed at each stage of a third-order RungeKutta method. In the simulations reported here, the second fractional step is integrated analytically. In Fourier space, (48)-(50) become

$$
\begin{gathered}
\frac{\partial \hat{\rho}}{\partial t}+i k_{l} \hat{m}_{l}=0 \\
\frac{\partial \hat{m}_{l}}{\partial t}+i k_{l} \hat{p}=0 \\
\frac{\partial \hat{p}}{\partial t}+i c_{0}^{2} k_{l} \hat{m}_{l}=0
\end{gathered}
$$

where $m_{l}=\rho v_{l}$ and Fourier transformed quantities (which depend upon the wavenumber $\mathbf{k}$ ) are denoted by a circumflex. The exact solution of these equations is

$$
\hat{\rho}^{(2)}=\hat{\rho}^{(1)}+\frac{1}{c_{0}^{2}}\left[\hat{A} \cos \left(c_{0} k \Delta t_{s}\right)+\hat{B} \sin \left(c_{0} k \Delta t_{s}\right)-\hat{A}\right]
$$




$$
\begin{gathered}
\hat{m}_{l}^{(2)}=\hat{m}_{l}^{(1)}-\frac{i k_{l}}{c_{0} k}\left[\hat{A} \sin \left(c_{0} k \Delta t_{s}\right)-\hat{B} \cos \left(c_{0} k \Delta t_{s}\right)+\hat{B}\right] \\
\hat{p}^{(2)}=\hat{A} \cos \left(c_{0} k \Delta t_{s}\right)+\hat{B} \sin \left(c_{0} \Delta t_{s}\right)
\end{gathered}
$$

where $k=|\mathbf{k}|$ is the magnitude of the Fourier wavenumber, $\hat{A}=\hat{p}^{(1)}, \hat{B}=i \frac{c_{0}}{k} k_{l} \hat{m}_{l}^{(1)}$. The superscript 1 denotes the result of the first fractional step of the splitting and the superscript 2 the results of the second fractional step. The effective time-step of the Runge-Kutta stage is denoted by $\Delta t_{s}$. The advantage of this splitting is that the principal terms responsible for the acoustic waves have been isolated. Since they are treated semi-implicitly, one expects the time-step limitation to depend upon $v+\left|c-c_{0}\right|$ rather than $v+c$. (Although there is also a viscous stability limit for the first fractional step, it is well below the advection limit in the cases of interest.) This is clearly a substantial advantage at low Mach numbers. Since the second fractional step is integrated analytically, it does not contribute to any time step limitations. If one is truly interested in all the details arising from the sound waves, or if there is a substantial coupling between the sound waves and the rest of the flow, then the time-step must be small enough to resolve the temporal evolution of these waves. But, if only the larger-scale sound waves are of interest, then this splitting method is useful.

During the acoustic fractional step, an isotropic truncation is performed: for each variable $(\rho, \rho \mathbf{v}$ and $p$ ), all Fourier coefficients for which

$$
k_{l} k_{l} \geq(N / 2)^{2}
$$

are set to zero. This reduces the numerical anisotropy produced by a cubic truncation. Moreover, it reduces the aliasing interactions in the collocation method (Canuto et al 1987, Chapters 3 and 7).

The compressible code can also be executed in a purely explicit mode. In this case no splitting is performed; Eqs. (45)-(50) are simply combined in the appropriate manner and integrated directly.

The expected stability limit of this three-dimensional Fourier collocation method for the compressible Navier-Stokes equations has the form

$$
\Delta t<\alpha\left[\max _{g r i d} \sum_{i=1}^{3} \mathcal{U}_{i}\right]^{-1}\left(\frac{2 \pi}{N}\right)
$$

where for the semi-implicit version,

$$
\mathcal{U}_{i}=\left|v_{i}\right|+\left|c-c_{0}\right|
$$

while

$$
U_{i}=\left|v_{i}\right|+|c|
$$

when the time advancement is fully explicit. For the third-order Runge-Kutta method employed here, we use $\alpha=0.5$.

A number of simulations have also been conducted of strictly incompressible flow. These were performed with a separate code which also used a Fourier collocation method, but for the simpler, incompressible Navier-Stokes equations. 


\section{Comparison with Incompressible Results}

The initial conditions for the numerical simulations were designed to reproduce the experimental data of Comte-Bellot and Corrsin (1971) on isotropic turbulence, hereafter referred to as CBC. These experiments were also the basis of direct simulations used by Clark, Ferziger and Reynolds (1979), Bardina, Ferziger and Reynolds (1983), and McMillan and Ferziger (1979) in their analyses of incompressible LES models. Initial conditions are chosen to match CBC measurements at a non-dimensional time of 240 (cf. table 4 in Comte-Bellot and Corrsin 1971). The computational domain is a cube of side $20 / 2 \pi \mathrm{cm}$. The CBC parameters are associated with measurements taken behind a grid with a mesh spacing of one inch, and a mean fluid velocity of $393.7 \mathrm{in} / \mathrm{sec}$. The initial time in the direct simulation corresponds to $t=0.00254 \mathrm{sec}$ in the CBC experiment. The reference length $\left(L_{0}\right)$, velocity $\left(U_{0}\right)$ and pressure $\left(P_{0}\right)$ are respectively $20 / 2 \pi \mathrm{cm}, 1 \mathrm{~cm} / \mathrm{sec}$ and $1 \mathrm{gr} / \mathrm{cm} \mathrm{sec}^{2}$. After generating a random, divergence-free velocity profile, the kinetic energy (in Fourier space) is scaled to match the measured CBC energy spectrum (see Appendix). Finally, the velocities are scaled so that the initial rms velocity agrees with the measured values. This adjustment is typically less than $1 \%$, which provides one measure of the uncertainty in the fit to the experimental data. With the chosen non-dimensionalization, the Reynolds number $R e=U_{0} L_{0} / \nu$ is 22.74 based on a kinematic viscosity $\nu=0.14 \mathrm{~cm}^{2} / \mathrm{sec}$. Table 1 summarizes the parameters measured

\begin{tabular}{|c|c|c|c|c|}
\cline { 2 - 5 } \multicolumn{1}{c|}{} & CBC & $64^{3}$ & $96^{3}$ & $128^{3}$ \\
\hline $\mathbf{v}_{\mathbf{r m}}$ & 6.75 & 6.75 & 6.75 & 6.75 \\
$\frac{1}{3} \operatorname{tr}(\mathbf{S k})$ & - & 0.0 & 0.0 & 0.0 \\
$E$ & - & 68.3 & 68.3 & 68.3 \\
$\epsilon$ & 462 & 375 & 432 & 447 \\
$\lambda_{11}$ & 0.26 & 0.28 & 0.27 & 0.25 \\
$\lambda_{12}$ & - & 0.20 & 0.19 & 0.27 \\
$\lambda_{13}$ & - & 0.20 & 0.19 & 0.26 \\
$R_{\lambda}$ & 38.1 & 43.6 & 41.3 & 37.8 \\
\hline
\end{tabular}

Table 1: Initial conditions based on CBC experiment and Clark et al. (1979) calculation. Mach number is zero.

by CBC at $t=240$. The Taylor microscale length $\lambda_{k l}$ is defined by

$$
\lambda_{k l}=\left[\frac{\left\langle v_{k}^{2}\right\rangle}{\left\langle\left(\frac{\partial v_{k}}{\partial x_{l}}\right)^{2}\right\rangle}\right]^{1 / 2}
$$


and the dissipation $\epsilon$ by

$$
\epsilon=2 \mu \int S_{i j} S_{i j} d^{3} x
$$

where $S_{i j}$ is the rate of strain tensor

$$
S_{i j}=\frac{1}{2}\left(\frac{\partial v_{i}}{\partial x_{j}}+\frac{\partial v_{j}}{\partial x_{i}}\right)
$$

In Eq. (62), $\langle\cdot\rangle$ denotes a spatial average. Its exact definition is given in the Appendix. The Taylor microscale Reynolds number is

$$
R_{\lambda}=\frac{v_{1} \lambda_{11}}{\nu}
$$

The velocity derivative skewness and flatness tensors Sk and Fl are the third and fourth moments of the velocity gradient and are defined by

$$
\begin{gathered}
S k_{i j}=\frac{\left\langle\left(\frac{\partial v_{i}}{\partial x_{j}}\right)^{3}\right\rangle}{\left\langle\left(\frac{\partial v_{i}}{\partial x_{j}}\right)^{2}\right\rangle^{3 / 2}} \\
F l_{i j}=\frac{\left\langle\left(\frac{\partial v_{i}}{\partial x_{j}}\right)^{4}\right\rangle}{\left\langle\left(\frac{\partial v_{i}}{\partial x_{j}}\right)^{2}\right\rangle^{2}} .
\end{gathered}
$$

In tables 1 and 2 , only the trace of the skewness and flatness tensors are shown. The remaining columns list the parameters obtained from the initial conditions of the numerical simulations on $64^{3}, 96^{3}$ and $128^{3}$ grids. There is a $20 \%$ discrepancy between the dissipation obtained by CBC and the dissipation computed on the coarsest grid which suggests that a $64^{3}$ grid has marginal resolution, at best. A $12 \%$ difference between the value of $R_{\lambda}$ calculated on the $64^{3}$ grid and that obtained by CBC confirms the need for grids finer than $64^{3}$. On a $96^{3}$ grid, both the dissipation and $R_{\lambda}$ are in much closer agreement with CBC. Discrepancies between our results and CBC for $\epsilon$ and $R_{\lambda}$ are respectively $6.5 \%$ and $7.5 \%$ on a $96^{3}$ grid. On the finest grids on which the direct simulations were performed, the computed values of $\epsilon$ and $R_{\lambda}$, respectively, have relative errors of $3.5 \%$ and less than $1 \%$ when compared to CBC.

The numerical simulations were run from $t=240$ until $t=375$ (in CBC units), which corresponds to a non-dimensional time interval of 0.1145 (in our units). Table 2 furnishes a comparison of the experimentally measured parameters with those from the numerical simulation at the final time. On the coarsest grid, the total dissipation rate that was calculated is still slightly below the value measured by CBC. A $96^{3}$ grid generates values of $\epsilon$ consistent with CBC. 


\begin{tabular}{|c|c|c|c|c|}
\cline { 2 - 5 } \multicolumn{1}{c|}{} & CBC & $64^{3}$ & $96^{3}$ & $128^{3}$ \\
\hline $\mathbf{v}_{r m s}$ & 5.03 & 5.18 & 5.19 & 5.21 \\
$\frac{1}{3} \operatorname{tr}(\mathbf{S k})$ & - & -0.42 & -0.51 & -0.52 \\
$E$ & 38.6 & 40.3 & 40.4 & 40.7 \\
$\epsilon$ & 154.4 & 151.3 & 154.6 & 156.8 \\
$\lambda_{11}$ & 0.34 & 0.33 & 0.34 & 0.33 \\
$\lambda_{12}$ & - & 0.24 & 0.24 & 0.23 \\
$\lambda_{13}$ & - & 0.24 & 0.24 & 0.23 \\
$R_{\lambda}$ & 36.6 & 37.8 & 40.4 & 37.2 \\
\hline
\end{tabular}

Table 2: Final conditions $(t=0.1145)$ based on CBC experiment and Clark et al. (1979) calculation. Mach number is zero.

At $t=0.1145$, the diagonal components of $\mathbf{S k}$ are -0.5 which agree well with the numerical results of Kerr (1985). Kerr studied isotropic, turbulent flow, but prevented the decay of energy by using an exterior energy source at the large length scales.

As noted in the previous section, we have chosen not to de-alias the advection terms. In reaching this decision we drew upon the extensive evidence that has accumulated on aliasing effects in the last dozen years (Canuto, et al 1987., Chapters 3, 4 and 7) and upon tests conducted with the incompressible isotropic turbulence code. In this code, de-aliasing is accomplished by applying the 2/3-rule (Canuto, et al 1987, Chapters 3 and 7 ) in an isotropic fashion; e.g., the de-aliased results for a $64^{3}$ grid are obtained by running the incompressible code on a $96^{3}$ grid and applying the truncation given by (58) with $N / 3$ in place of $N / 2$ on the right hand side. The results are summarized in Fig. 1. Here we present the energy spectra $E(k)$ (defined in the Appendix) for $64^{3}, 96^{3}$, and $128^{3}$ grids at $t=0.0586$ for both aliased and de-aliased calculations. Some adverse effects of aliasing are apparent on the $64^{3}$ grid, but they are only in the tail of the spectra, and they are already insignificant on a $96^{3}$ grid. For the reasons outlined here, a $96^{3}$ grid was chosen as the standard discretization for the incompressible and for the compressible simulations.

\section{Compressible Turbulence Results}

\subsection{Direct Simulations}

Recent work on the direct numerical simulation of homogeneous compressible turbulence has indicated the crucial role played by the initial conditions. Passot and Pouquet (1986) conducted direct simulations of two-dimensional, compressible isotropic turbulence and concluded that when the initial rms density fluctuations are small, the turbulence statistics 
remain quasi-incompressible for turbulent Mach numbers $M_{t}$ less than 0.3.

$$
M_{t}=M_{\infty}\left\langle\frac{v^{2}}{T}\right\rangle^{1 / 2}
$$

They also demonstrated (through the use of direct numerical simulations) that eddy shocklets result for sufficiently high initial rms density fluctuations and/or turbulent Mach numbers. A more systematic analysis and categorization of the effect of the initial conditions on compressible isotropic turbulence was achieved recently by Erlebacher et al. (1990).

They concluded that for $0<M_{t}<0.3, p_{\text {rme }}$ must initially be $O\left(M_{t}\right)$ for the resulting turbulence statistics to become strongly compressible with an $O(1)$ ratio of compressible to incompressible turbulent kinetic energy. (For the range of $M_{t}$ considered herein, no eddy-shocklets occur.) On the other hand, if initially $\rho_{\text {rmes }}, T_{r m o} \ll M_{t}$, then the resulting turbulence statistics remain quasi-incompressible.

We first present the results of direct numerical simulations of compressible isotropic turbulence corresponding to the initial conditions of the $\mathrm{CBC}$ experiment but with a variety of non-zero mean Mach numbers. Since for these simulations, the initial conditions are $\rho_{\text {rms }}=0, T_{r m s} \ll 1$, only weakly compressible turbulence statistics are expected according to the theoretical results of Erlebacher et al. (1990). Unless specified otherwise, a subscript rms for any variable $\mathcal{F}$ refers to the quantity $\left\langle(\mathcal{F}-\langle\mathcal{F}\rangle)^{2}\right\rangle^{1 / 2} /\langle\mathcal{F}\rangle$.

The initial pressure distribution over the entire field is specified. The fluctuating pressure, $p_{f}$ is determined from the velocity distribution by enforcing a zero initial time derivative for $\nabla$.v. A Poisson equation for $p_{f}$ is obtained from the divergence of the momentum equation after setting the time variation of $\boldsymbol{\nabla} \cdot \mathbf{v}$ to zero (Feiereisen et al. 1981). The mean pressure, $p_{m}$, is then determined so that a prescribed initial mean average Mach number, $M_{0}$, defined to be the ratio of $\mathrm{rms}$ fluid velocity and $\mathrm{rms}$ speed of sound, is achieved. An analytic expression for $p_{m}$ is given by

$$
p_{m}=\frac{M_{0}^{2} \int v^{2} d^{3} x+\int \gamma p_{f} \rho^{-1} d^{3} x}{\gamma \int \rho^{-1} d^{3} x} .
$$

The initial average Mach number is specified at the outset of the direct numerical simulations (DNS) as an initial condition. Density is initially set to unity, while the temperature, if required, is derived from the equation of state. Direct numerical simulations are performed for $M_{0}=0.0,0.1,0.4$ and 0.6 .

The Mach 0.6 case contains localized regions of supersonic flow as evidenced by tables 3-4 and by the three-dimensional contour of Mach 1 furnished in figure 2. Nonetheless, the statistical properties of the flow remain largely unaffected by compressibility effects. This is shown in figures 3-6 which track the time histories of several statistical variables obtained from $96^{3}$ DNS. The time histories for skewness (fig. 3), $\lambda_{11}$ (fig. 4), and total kinetic energy (fig. 5) at Mach numbers $0.0,0.1,0.4$ and 0.6 are virtually superimposed on each other.

Flatness and skewness are affected the most by compressibility effects in these simulations. Figure 3 indicates that the skewness which corresponds to an isotropic turbulent state 
monotonically increases with Mach number. It is -0.50 at $M_{0}=0$ and has increased to -0.46 at $M_{0}=0.6$. Before the flow has reached a state of isotropic turbulence, the time evolution of skewness at all Mach numbers are indistinguishable from each other. The physical system has equilibrated after approximately one third the total computation time. While not reaching an equilibrium value, it is nonetheless worthwhile to point out that the flatness parameter decreases by $2 \%$ as the Mach number is raised from 0.0 to 0.6 as seen in figure 6 .

Figure 5 illustrates the decay of turbulent kinetic energy $\left(\int \frac{1}{2} v_{i} v_{i} d^{3} x\right)$ as a function of time. This decay is a natural consequence of viscous damping. After a brief initial increase, $R_{\lambda}$ continuously decreases in time, (fig. 7), with no sign of stabilizing. On the other hand, $\lambda$ which is representative of the smaller eddies, increases in time (fig. 4). This indicates that energy in the higher wavenumbers is being depleted by the molecular viscosity.

Tables 3-5 summarize the results of direct simulations of compressible isotropic turbulence for $M_{0}=0.1,0.4$ and 0.6 , for $t=0.1145$ on three different grids T. Incompressible results are included for comparison. On all the grids, the compressible data converges to the in-

\begin{tabular}{|c|c|c|c|c|c|c|}
\hline$M_{0}$ & $E$ & $\epsilon$ & $|\nabla \cdot \mathbf{v}|_{\max }$ & $\left\langle\frac{1}{3} \operatorname{tr}(\mathbf{S k})\right\rangle$ & $\langle M\rangle$ & $M_{\max }$ \\
\hline 0.0 & 40.26 & 157.4 & 0.00 & -0.424 & 0.00 & 0.00 \\
0.1 & 40.82 & 158.2 & 0.17 & -0.440 & 0.07 & 0.21 \\
0.4 & 41.09 & 160.4 & 1.50 & -0.428 & 0.28 & 0.84 \\
0.6 & 41.32 & 162.3 & 3.30 & -0.406 & 0.43 & 1.26 \\
\hline
\end{tabular}

Table 3: Summary of direct simulations on a $64^{3}$ grid with initial average Mach numbers of $0.0,0.1,0.4$ and 0.6 at $t=0.1145$.

\begin{tabular}{|c|c|c|c|c|c|c|}
\hline$M_{0}$ & $E$ & $\epsilon$ & $|\nabla \cdot \mathbf{v}|_{\max }$ & $\left\langle\frac{1}{3} \operatorname{tr}(\mathbf{S k})\right\rangle$ & $\langle M\rangle$ & $M_{\max }$ \\
\hline 0.0 & 40.35 & 154.3 & 0.00 & -0.506 & 0.00 & 0.00 \\
0.1 & 40.49 & 155.5 & 0.14 & -0.505 & 0.07 & 0.23 \\
0.4 & 40.79 & 157.0 & 1.17 & -0.493 & 0.28 & 0.93 \\
0.6 & 41.04 & 158.3 & 2.82 & -0.477 & 0.42 & 1.39 \\
\hline
\end{tabular}

Table 4: Summary of direct simulations on a $96^{3}$ grid with initial average Mach numbers of $0.0,0.1,0.4$ and 0.6 at $t=0.1145$.

compressible results as the Mach number is driven towards zero. As expected, the divergence of velocity no longer vanishes, and is now an increasing function of $M_{0}$.

\footnotetext{
"A DNS at $M_{0}=0.6$ was not performed on the $128^{3}$ grid.
} 


\begin{tabular}{|c|c|c|c|c|c|c|}
\hline$M_{0}$ & $E$ & $\epsilon$ & $|\nabla \cdot \mathbf{v}|_{\max }$ & $\left(\frac{1}{3} \operatorname{tr}(\mathbf{S k})\right\rangle$ & $\langle M\rangle$ & $M_{\max }$ \\
\hline 0.0 & 40.29 & 153.98 & 0.00 & -0.521 & 0.00 & 0.00 \\
0.1 & 40.39 & 154.84 & 0.14 & -0.518 & 0.07 & 0.21 \\
0.4 & 40.78 & 156.54 & 1.11 & -0.505 & 0.28 & 0.86 \\
\hline
\end{tabular}

Table 5: Summary of direct simulations on a $128^{3}$ grid with initial average Mach numbers of $0.0,0.1$ and 0.4 at $t=0.1145$

While the dissipation is approximately the same on the two finer grids, the consistently lower values on the coarsest grid confirm the previously stated conclusion that a $64^{3}$ grid cannot resolve all the length scales. As a function of increasing Mach number, the trace of Sk increases, the dissipation decreases, while the total kinetic energy increases very slightly.

The results in tables 3-5 are averages over several DNS runs with different initial seeds. A given seed uniquely determines the initial velocity distribution, and therefore the pressure and temperature fields. Variations of the seed are only necessary to eliminate the statistical uncertainty due to the random velocity distribution. The distribution of velocity on two different grid sizes are different even when the initial seed is the same.

Skewness is even more sensitive to the grid refinement than the dissipation as witnessed by its decrease from a value of -0.505 to one of -0.521 on $96^{3}$ and $128^{3}$ grids respectively. This might be a result of the greater sensitivity of the fluctuating velocity field spatial derivatives to slight inaccuracies in the flow variables.

\subsection{Data Analysis}

Using the data generated from the previously discussed DNS of compressible homogeneous turbulence at low Mach numbers, the proposed subgrid-scale (SGS) model is now validated. Models relate the subgrid-scale stresses - which are not available to a large-eddy simulation code - to the large scale velocities which are known. These velocities are simply the Favrefiltered velocities introduced earlier. The Favre-filtered velocities are calculated by filtering the resolved DNS velocity field with a Gaussian spatial filter of width $\Delta=\Delta_{f} \Delta x_{f}$, where $\Delta x_{f}$ is the grid spacing on the fine grid. For convenience, $\Delta_{c}$ and $\Delta_{f}$ refer to the filter width $\Delta$ non-dimensionalized with respect to the coarse and fine grid spacing respectively.

Perturbed velocity fluctuations on the fine grid are the difference between the fully resolved velocity and the filtered ones, given by

$$
\mathbf{v}^{\prime}=\mathbf{v}-\tilde{\mathbf{v}} .
$$

From $\mathbf{v}^{\prime}$ and $\tilde{\mathbf{v}}$, subgrid-scale stresses based on DNS, refered to as exact, are calculated. 
These include the Leonard, cross and Reynolds subgrid-scale stresses given by Eqs. (20)(22). However, these subgrid-scale stresses themselves do not directly affect the evolution of the system. The momentum equation is only influenced by the divergence of the subgrid-scale stresses (i.e., the vector level). Similarly, $\tilde{\mathbf{v}} \cdot(\nabla \cdot \tau)$ (the scalar level) is a better representation of the dissipation terms in the energy equation than are the stresses. Consequently, correlations are performed on the tensor, vector and scalar levels. Ideally, high correlations are desired on all levels.

The data analysis proceeds in multiple stages. First, the exact stresses calculated from the DNS are injected down to the coarse grid, along with the filtered velocities. The modeled subgrid-scale stresses are then calculated (excluding the model constants) on the coarse grid. Some variables must be filtered a second time (e.g. cross stress terms). Rather than calculate them on the fine grid (which is not available to the large-eddy simulation codes), a Gaussian filter is applied to $\overline{\mathbf{v}}$ on the coarse grid with a filter width of $\Delta_{c}$. Consistency between the coarse and fine filter widths is achieved by insuring that

$$
\frac{\Delta_{f}}{\Delta_{c}}=\frac{N_{f}}{N_{c}}
$$

where $N_{f}$ and $N_{c}$ are respectively the number of nodes along one direction of the fine and coarse grids. This guarantees that the filtering on the coarse and fine grids is performed over the same region in physical space. Derivative evaluations on both the coarse and the fine grid are based on Fourier collocation. Calculations by McMillan and Ferziger (1979) indicate that the model constants are sensitive to the accuracy of the derivative evaluations. A general trend that has been observed is that the Smagorinsky constant is lowered when derivative quantities are evaluated more accurately. Our constants are therefore expected to lie in the lower range of the values obtained by McMillan (1980).

Next, the model constant, $C_{R}$, is calculated. Unfortunately, the constants can be calculated by a wide variety of algorithms, each with its own merits. Moreover, for each algorithm, the constants can be evaluated from tensor, vector or scalar information. Therefore, criteria must be established to identify the best method. A key test is that $\mathbf{L}+\mathbf{C}$ should be Galilean invariant. To make use of this fact, an additional constant, $C_{C}$, is introduced as an extra factor in the subgrid-scale cross stress model. A self-consistent method of calculating the constants must reproduce $C_{C}=1$ to satisfy the Galilean invariance property stated above (Speziale 1985). Additional tests are performed on coarse grids with varying degrees of refinement which further decrease the number of choices. A thorough discussion of model constants is the subject of the next subsection.

Once a single or a multiple set of model constants have been determined, the model subgrid-scale stresses are calculated and correlated with the exact subgrid-scale stresses calculated from the DNS after injection onto the coarse grid. The correlations are performed for each type of subgrid-scale stress individually, and for the total stress $(L+C+R)$. Strong differences in the correlation coefficients relating total stresses are noticed depending on whether or not the Leonard stresses are included. Finally, the correlations obtained from the proposed model are compared with the linear combination model, which has been shown 
to be one of the best models available for incompressible isotropic turbulence. Correlation coefficients are calculated based on the two pairs of constants that are obtained from the above considerations. The set that is finally retained corresponds to the highest levels of correlation of the total stress on the vector and scalar levels. These matters are treated more completely in a later subsection.

To avoid a possible confusion of terminology when referring to variables being compared against each other, superscripts $m$ and $e$ are sometimes used. They respectively refer to modeled and exact (based on DNS) variables at the tensor, vector and scalar levels.

\subsubsection{Model Constants}

The proposed model given by equations (31) and (42) has two undetermined coefficients. The constant, $C_{R}$, is associated with the modeled subgrid-scale Reynolds stress, $\mathbf{R}$, while $P r_{T}$ is associated with the thermal heat flux.

Although the cross stress model has no constant associated with it, it is nonetheless multiplied by a constant $C_{C}$. This is done in the hope of reducing the number of schemes by which the constants can be calculated. A good model should reproduce a cross stress constant of one to guarantee Galilean invariance. Once the constants have been determined, $C_{C}$ is set to one and forgotten. Because the flow is isotropic, constants are expected to be the same for the three diagonal stress components, the three off-diagonal components and the three vector components. Therefore, the values presented in the tables below are averaged over the appropriate components. In the tables, $D$ refers to averaged diagonal components, $O D$ to averaged off-diagonal components, $V$ to averaged vector components and $S$ to averaged scalar components. Similar averagings are performed for the correlation coefficients.

The two constants $\left(C_{R}\right.$ and $\left.C_{C}\right)$ are calculated using two techniques - each applied on the tensor, vector and scalar levels. One method enforces equality of the rms levels of the exact and modeled stresses. This is done for each individual subgrid-scale stress (i.e., the subgrid-scale Reynolds stress and the cross stress). Hereafter this approach is referred to as RMS. The second method utilizes multiple linear least square regression (LSQ) between the exact (18) and the modeled (31) total subgrid-scale stresses to determine the constants. Table 6 summarizes the results obtained from incompressible data. The three cases presented are identical except for the initial random number seed. The constants are independent of the detailed velocity statistics. These results are based on a vector level comparison between the modeled and exact stresses. Both RMS and LSQ produce $C_{C}$ close to unity as required. Unfortunately this prevents a rational choice from being made between the two approaches. A more complete set of LSQ constants is presented in table 7. They are computed at Mach numbers of $0.0,0.1,0.4$ and 0.6 on the tensor, vector and scalar level. Computations are performed on a coarse grid of $16^{3}$. 


\begin{tabular}{|c|c|c||c|c|}
\cline { 2 - 5 } \multicolumn{1}{c|}{} & \multicolumn{2}{c||}{ LSQ } & \multicolumn{2}{c|}{ RMS } \\
\hline Seed & $C_{R}$ & $C_{C}$ & $C_{R}$ & $C_{C}$ \\
\hline 1 & 0.012 & 1.04 & 0.023 & 1.03 \\
2 & 0.012 & 1.03 & 0.022 & 1.04 \\
3 & 0.012 & 1.03 & 0.022 & 1.03 \\
\hline
\end{tabular}

Table 6: Model constants calculated by LSQ and RMS between exact and modeled total stresses $(\mathbf{L}+\mathbf{C}+\mathbf{R})$. Results are based on three identical incompressible simulations except for the random initial velocity distributions. Calculations are on the vector level on a $16^{3}$ grid.

At first glance, $C_{C}$ is near unity at both the scalar and vector levels. However whereas on the vector level, the constant remains within $4 \%$ of unity for all Mach numbers, this is not the case on the scalar level where $C_{C}$ is a decreasing function of Mach number. This trend is present in the data generated from both random seeds. Although not presented here, the rms cross stress model constant is also near unity when calculated based on vector level stresses. Therefore, a preferred method for the determination of the model constants is still not possible.

\subsubsection{Filter Width and Grid Coarseness}

Confirmation of the numerical evidence presented by McMillan and Ferziger (1979) that $\Delta_{c}=2$ is the best filter width is given in table $8\left(M_{0}=0.1\right)$. The criterion used to determine the validity of the filter width is that $C_{C}^{R M S}$ must remain close to unity on the vector level. Only when $\Delta_{c}=2$ is $C_{C}$ near one. Similar results hold for $L S Q$ constants. The constants also vary with respect to the coarse grid on which the LES is to be performed. Table 9 summarizes the model LSQ and RMS constants evaluated from modeled stresses calculated on $16^{3}$ and $32^{3}$ grids on the vector level. The data $\left(M_{0}=0.1\right)$ shows that $C_{R}$ varies by $30 \%$ when the grid size on which the modeled stresses are calculated ranges from $16^{3}$ to $32^{3}$. On the other hand, large eddy simulations using finite-difference algorithms might be performed on grids as large as $128^{3}$. Unless a subgrid-scale model is found which produces constants independent of the coarse grid size, LES simulations will run the risk of producing the wrong results. Perhaps a more complicated dependence of the modeled subgrid-scale Reynolds stresses on $\Delta$ is required.

The best model constants will produce the highest correlations between the modeled and exact subgrid-scale stresses on all levels (tensor, vector and scalar). Based on the previous discussion, only constants calculated on a vector level are adequate because they produce a $C_{C}$ of unity. Unfortunately, a clear cut choice between RMS and LSQ constants cannot be made because $C_{C}$ (on the vector level) is nearly one in both cases. Rather than make 
an arbitrary choice, both sets of constants are considered when correlating exact against modeled stresses. For reference, the constants used henceforth are

$$
\begin{aligned}
C_{R}^{L S Q} & =0.012 \\
C_{R}^{R M S} & =0.023 .
\end{aligned}
$$

\begin{tabular}{|c|c|c|c|c|c||c|c|}
\cline { 2 - 8 } \multicolumn{2}{c|}{} & \multicolumn{9}{c||}{ Seed 1 } & \multicolumn{2}{c|}{ Seed 2 } \\
\hline$M_{0}$ & & 0.0 & 0.1 & 0.4 & 0.6 & 0.0 & 0.6 \\
\hline & $D$ & 1.32 & 1.32 & 1.32 & 1.31 & 1.32 & 1.31 \\
$C_{C}$ & $V$ & 1.04 & 1.04 & 1.02 & 1.00 & 1.04 & 1.00 \\
& $S$ & 1.00 & 1.00 & 0.95 & 0.873 & 0.971 & 0.934 \\
\hline & $D$ & 0.018 & 0.018 & 0.018 & 0.018 & 0.016 & 0.015 \\
$C_{R}$ & $V$ & 0.012 & 0.012 & 0.012 & 0.012 & 0.012 & 0.012 \\
& $S$ & 0.015 & 0.015 & 0.015 & 0.014 & 0.015 & 0.015 \\
\hline
\end{tabular}

Table 7: LSQ model constants. Filter widths are $\Delta_{f}=12$ and $\Delta_{c}=2$.

\begin{tabular}{|c|c|c|c||c|c|c|}
\cline { 2 - 7 } \multicolumn{1}{c|}{} & \multicolumn{3}{c||}{ LSQ } & \multicolumn{3}{c|}{ RMS } \\
\hline$\triangle_{f}$ & 6 & 12 & 24 & 6 & 12 & 24 \\
$\Delta_{c}$ & 1 & 2 & 4 & 1 & 2 & 4 \\
\hline$C_{R}$ & 0.007 & 0.012 & 0.020 & 0.019 & 0.023 & 0.034 \\
$C_{C}$ & 0.31 & 1.03 & 1.33 & 0.82 & 1.03 & 1.13 \\
\hline
\end{tabular}

Table 8: LSQ and RMS model coefficients between exact and modeled stresses on the vector level at $M_{0}=0.1$. Results are obtained with fine filter widths of 6,12 and 24 while maintaining the proper ratio of 6 between fine and coarse widths. The coarse grid is $16^{3}$.

\subsubsection{Correlations}

Correlation coefficients have long been a preferred diagnostic tool for estimating the reliability of the modeled stresses. However, the subgrid-scale stresses have been separated into various components ( $\mathbf{L}, \mathbf{C}, \mathbf{R})$, each modeled separately and although the correlation of any of these components against their models might be excellent, it is still possible for the correlation of the exact total stress against the modeled total stress to be less impressive. Such is the case, for instance, when two stress components have opposite signs and partially cancel each 


\begin{tabular}{|c|c|c||c|c|}
\cline { 2 - 5 } \multicolumn{1}{c|}{} & \multicolumn{2}{c||}{ LSQ } & \multicolumn{2}{c|}{ RMS } \\
\hline Grid & $C_{R}$ & $C_{C}$ & $C_{R}$ & $C_{C}$ \\
\hline $16^{3}$ & 0.0125 & 1.03 & 0.023 & 1.03 \\
$32^{3}$ & 0.0094 & 1.03 & 0.013 & 1.02 \\
\hline
\end{tabular}

Table 9: LSQ and RMS model constants based on subgrid-scale stresses evaluated on $16^{3}$ and $32^{3}$ coarse grids.

other out. As a final note, before the specific correlation coefficients are presented, one must always be attentive to the actual relationship between the exact and the modeled variable, even when the correlation coefficient is relatively high (say, above 70\%). A correlation coefficient as high as $70 \%$ may not be as good as it seems. For example, the correlation between the functions $y=x$ and $y=\exp (-x)$ in the interval $[0,1]$ is approximately $70 \%$ although they are qualitatively different functions! As a consequence, correlations are only deemed good if the correlation coefficient is above, say, the $90 \%$ level.

For convenience, the compressible subgrid-scale model is restated here:

$$
\begin{gathered}
{ }_{D} R_{i j}=2 C_{R} \bar{\rho} \Delta^{2}\left(\tilde{S}_{k l} \tilde{S}_{k l}\right)^{1 / 2}\left(\tilde{S}_{i j}-\frac{1}{3} \tilde{S}_{m m} \delta_{i j}\right) \\
{ }_{I} R_{i j}=0 \\
C_{i j}=-\bar{\rho}\left(\tilde{v}_{i} \tilde{v}_{j}-\tilde{\tilde{v}}_{i} \tilde{\tilde{v}}_{j}\right)
\end{gathered}
$$

The correlation coefficients presented in table 10 between exact and modeled $\mathbf{R}$ and $\mathbf{C}$, are independent of the model constants. The correlation coefficients are insensitive to the average Mach number variation. The neglect of ${ }_{I} R_{i j}$ appears to be a good approximation; the direct simulations show it to be several orders of magnitude smaller than the thermodynamic pressure. For example, for all of the compressible isotropic turbulence simulations conducted in this study,

$$
\frac{\left(\nabla \cdot{ }_{I} \mathbf{R}\right)_{r m s}}{(\nabla p)_{r m s}}<3 \times 10^{-3}
$$

and, hence, the effect on the isotropic part of the Reynolds stress tensor is dominated by the thermodynamic pressure.

In most of the literature on subgrid-scale models, the Leonard stress has been omitted from the total stress correlations on the grounds that it is calculated exactly (Bardina, Ferziger, and Reynolds 1983, McMillan 1980, McMillan and Ferziger 1979). However, it has recently been shown by Speziale (1985) that the combination $\mathbf{L}+\mathbf{C}$ is Galilean invariant, while the Leonard and cross stresses, individually, are not. Therefore we feel that correlations of the total stress should include the Leonard stress. Table 11 summarizes the correlation coefficients between the total stress including and excluding the Leonard stress. Results 


\begin{tabular}{|c|c|c|c|c|}
\hline$M_{0}$ & & 0.1 & 0.4 & 0.6 \\
\hline & $D$ & 31 & 31 & 31 \\
$\mathrm{R}$ & $O D$ & 26 & 26 & 25 \\
& $V$ & 22 & 22 & 22 \\
& $S$ & 45 & 45 & 45 \\
\hline & $D$ & 89 & 89 & 89 \\
$\mathrm{C}$ & $O D$ & 91 & 91 & 91 \\
& $V$ & 80 & 80 & 80 \\
& $S$ & 75 & 74 & 72 \\
\hline
\end{tabular}

Table 10: Correlations between exact and modeled stresses, $\mathbf{R}$ and $\mathbf{C}$, at Mach 0.1, 0.4 and 0.6.

are presented at Mach 0 and Mach 0.6. When the Leonard stresses are left out, correlation coefficients similar to those of Bardina are obtained on all levels. However, the inclusion of $\mathbf{L}$ decreases the correlations at the vector level by approximately $30 \%$. Table 11 substantiates that the correlation coefficients (with and without L) are nearly independent of the initial average Mach number.

Correlation coefficients between $(\mathbf{C}+\mathbf{R})^{e}$ and various combinations of the modeled stresses using constants calculated by LSQ and RMS are summarized in table 12. The second column indicates the model against which the total stress is being compared. Although at first glance RMS based constants perform better at the tensor level when the total modeled stress is considered, at the vector and scalar levels, the trend is reversed. Correlations at the vector and scalar level are higher by $4 \%$ using the LSQ constants.

When the constants are selected based on LSQ, table 12 indicates that the correlations on all levels are highest when all modeled components are included. However, the dynamic evolution of the large scale velocities only brings into play the stresses on the vector and scalar level. Therefore the coefficients which produce the maximum correlations of total stress on these two levels should be chosen. This leads to an optimum choice of

$$
C_{R}=0.012
$$

calculated by least squares fit of the total stress on the vector level.

Conversion of $C_{R}$ to the standard form currently used in incompressible LES " produces a Smagorinsky constant of 0.092 . McMillan (1979) obtained a value of $C_{S}=0.10$ when spectral collocation derivative computations were employed. This constant corresponds to a scalar level evaluation based on RMS. On the vector level, McMillan calculated a higher

\footnotetext{
"In a number of reports (McMillan and Ferziger 1979, Bardina et al. 1983), the subgrid-scale Reynolds stress model is proportional to $C_{S}^{2}$. In these cases, the relationship between $C_{S}$ and $C_{R}$ is $C_{R}=\sqrt{2} C_{S}^{2}$.
} 


\begin{tabular}{|c|c|c||c|c|}
\cline { 2 - 5 } \multicolumn{1}{c|}{} & \multicolumn{2}{c||}{$M_{0}=0.0$} & \multicolumn{2}{c|}{$M_{0}=0.6$} \\
\cline { 2 - 5 } \multicolumn{1}{c|}{} & $\mathrm{L}+\mathrm{C}+\mathrm{R}$ & $\mathrm{C}+\mathrm{R}$ & $\mathrm{L}+\mathrm{C}+\mathrm{R}$ & $\mathrm{C}+\mathrm{R}$ \\
\hline$D$ & 93 & 82 & 93 & 81 \\
$O D$ & 80 & 85 & 79 & 84 \\
$V$ & 46 & 72 & 46 & 71 \\
$S$ & 56 & 73 & 56 & 74 \\
\hline
\end{tabular}

Table 11: Comparison of correlation coefficients of the exact total stress versus its model. The modeled terms are computed on a $16^{3}$ coarse grid. Each case is presented with and without the inclusion of the Leonard subgrid-scale stress terms (calculated exactly). Both the incompressible and the $\mathrm{M}=0.6$ case are shown to illustrate the weak influence of Mach number on the correlation coefficients with $C_{R}=0.012$.

\begin{tabular}{|c|c|cccc||cccc|}
\cline { 3 - 9 } \multicolumn{2}{c|}{} & \multicolumn{4}{c||}{ Least squares } & \multicolumn{4}{c|}{ RMS } \\
\cline { 3 - 9 } \multicolumn{2}{c|}{} & \multicolumn{3}{c||}{$C_{R}=0.0122$} & \multicolumn{4}{c|}{$C_{R}=0.023$} \\
\hline Exact & Model & $D$ & $O D$ & $V$ & $S$ & $D$ & $O D$ & $V$ & $S$ \\
\hline C + R & $\mathbf{C}$ & 78 & 82 & 68 & 62 & 78 & 82 & 68 & 62 \\
& $\mathbf{C}+\mathbf{R}$ & 81 & 84 & 71 & 70 & 88 & 83 & 67 & 67 \\
\hline
\end{tabular}

Table 12: Correlation of the exact total stress $(\mathbf{C}+\mathbf{R})^{e}$ with various models. The modeled stresses are defined in equations (74)-(76).

Smagorinsky constant of 0.13 . This value can be obtained from the present data by using ${ }_{D} \mathbf{R}^{R M S}$ instead of ${ }_{D} \mathbf{R}^{L S Q}$. However as shown above, the correlations of the total subgridscale stress would be lower.

Initial tests of the subgrid-scale heat flux model produced a turbulent Prandtl number in the range of 0.4 to 0.5 . For this initial study, we take

$$
\operatorname{Pr}_{T}=0.5
$$

which is a value that has been used in previously published large-eddy simulations of turbulent flows with thermal convection (cf. Eidson 1985). A more accurate calculation of $\operatorname{Pr}_{T}$ could be accomplished in a compressible flow with significant mean temperature gradients; this is beyond the scope of the present study and must await future research. 


\section{Large-Eddy Simulation of Compressible Isotropic Turbulence}

Now, in order to demonstrate the efficacy of the subgrid-scale models derived in this paper, a large-eddy simulation of compressible isotropic turbulence is conducted. Since most highspeed compressible turbulent flows have significant regions where the turbulence statistics are quasi-incompressible, it is important that the models perform well for weakly compressible turbulence - the type of flow considered in the last section for the a priori tests. However, it is well known that a priori tests only provide a relatively weak gauge for the performance of subgrid-scale models in an actual large-eddy simulation (see Hussaini, Speziale and Zang 1990). It is therefore important to examine their performance in an actual large-eddy simulation, particularly for a case where significant compressibility effects are exhibited in the turbulence statistics.

Direct simulations of compressible isotropic turbulence were conducted corresponding to the initial conditions $R e=250,\left\langle M_{t}^{2}\right\rangle_{0}^{1 / 2}=0.1,\left(\rho_{\text {rms }}\right)_{0}=0,\left(T_{r m s}\right)_{0}=0.0626$ and two values of $\chi_{0}: 0$ and 0.2 which, respectively, correspond to initial values for $\left(R_{\lambda}\right)_{0}$ of 30.0 and 26.3 . Here, $\chi=E^{C} /\left(E^{I}+E^{C}\right)$ where $E^{I}$ and $E^{C}$ are the incompressible and compressible parts of the turbulent kinetic energy, respectively. The direct numerical simulations were performed on a $96^{3}$ mesh. Characteristic energy and dissipation spectra associated with the DNS are shown in figures 8 (a)-(c) and 9 (a)-(c) for $\chi_{0}=0$ and $\chi_{0}=0.2$, respectively. Figure 8 (b) clearly shows that $\chi$ remains very small for all time, with only very slight modification of the energy spectrum in time. In contrast, figure 9 (b) shows an initial cascade of the spectrum towards the smaller scales, followed by a strong energy dissipation at the smaller scales of $v_{i}^{C}$. The dissipation spectra $\left(k^{2} E(k)\right)$ shown in figures $8(\mathrm{a})$ and 9 (a) demonstrate that both the small and large scales are well resolved. Comparing figures 8 (c) and $9(\mathrm{c})$, the incompressible energy $E^{I}$ is the same at $t=0$ and $t=1.6$, but is influenced by compressibility effects at the intermediate times. This influence is characterized by a slight decrease in $E^{I}$ for $\chi_{0}>0$.

Integral properties of these two cases are plotted in figures 10 (a)-(e). These figures contrast the two runs corresponding to $\chi_{0}=0$ and $\chi_{0}=0.2$. The higher compressibility has a variety of effects on the flow. The total kinetic energy decays at a slightly slower rate, while both the skewness and the flatness are decreased. In other words, finite compressibility drives the flow more towards a Gaussian state. Both the integral scale $L_{I}$ and the Taylor microscale $\lambda_{11}$ become smaller as $\chi_{0}$ increases. Lastly, the decay rate of the microscale Reynolds number is slower for finite $\chi_{0}$. A more detailed study of these effects awaits future research. It would be particularly useful to compute these statistical quantities based on the solenoidal and irrotational components of velocity.

As noted by Frisch and Orszag (1990), the three dimensional vorticity is formed of tubular-like structures. This is graphically represented in figure 11 which shows a volume rendering of $\boldsymbol{\omega}^{2}$. Volumetric rendering is a visualization technique whereby rays are projected from the eye through the flow (which emits and absorbs light). The tubular structures of vorticity are contrasted with the spherical structure of $(\nabla \cdot \mathbf{v})^{2}$ shown in figure 12 . This difference is to be expected since the dilatation satisfies an isotropic wave equation to lead- 
ing order which shows no preferential direction. On the other hand, the vorticity stretching occurs along the axis of the vorticity vector, which then becomes a preferred direction.

The results for the direct simulation with the strongest compressibility effects $\left(\chi_{0}=0.2\right)$ were filtered and injected onto a coarse $32^{3}$ grid for comparison with an LES which was also performed on a $32^{3}$ grid using the subgrid-scale models derived herein. In this manner, a direct comparison can be made between the results of the LES and the direct simulation for a flow where the turbulence statistics exhibit significant compressibility. The following turbulence statistics were compared:

(1) the integrated average of $\omega_{i} \omega_{i}$ and $\left(\frac{\partial v_{i}}{\partial x_{i}}\right)^{2}$

(2) the integrated average total and isotropic vortex stretching (denoted by $\omega_{i} S_{i j} \omega_{j}$ and $\omega^{2} \nabla \cdot v$, respectively),

(3) the mean turbulence Mach number defined by $\left\langle M_{t}^{2}\right\rangle^{1 / 2}$

(4) the mean compressible, incompressible and total turbulent kinetic energy denoted by $E^{C}, E^{I}$ and $E_{T O T}$, respectively,

(5) the level of compressibility $\chi$ defined by $\chi=E^{C} / E_{T O T}$, and

(6) the rms of the thermodynamic pressure, density, and temperature denoted by $P_{r m s}$, $\rho_{r m s}$, and $T_{r m s}$, respectively.

These quantities represent a good choice of turbulence statistics to monitor the effects of compressibility.

In figure 13 (a)-(f), a direct comparison of these statistics for the DNS and LES is made for a filter width $\Delta_{f}=2$. It is clear that the LES does an excellent job in reproducing the results of the DNS with the possible exception of $\chi$. It should be noted that $\chi$ exhibits acoustic oscillations and hence is a difficult quantity to predict accurately; nonetheless, the LES yields results that are in good qualitative agreement with the direct simulation. The most striking result is how well the compressible turbulent kinetic energy and dilatational terms are captured.

It was found that a change in the filter width $\Delta_{f}$ to 1 or 3 - and an adjustment of the SGS model constants $C_{R}$ and $P r_{T}$ of up to $25 \%$ - only led to a mild degradation of the results. However, the subgrid-scale models did play a crucial role in obtaining the accurate results shown in figure 13 (i.e., a $32^{3}$ direct simulation is substantially under-resolved). To illustrate this point, the results of a direct simulation on the coarse $32^{3}$ grid (i.e., an LES for $\Delta_{f}=0$ ) is shown in figure 14 for the same test case. It is clear from this figure that the coarse grid DNS does a poor job in capturing the incompressible as well as the compressible turbulence statistics. Consequently, it is the adequate performance of the subgrid-scale 
models in draining the proper amount of energy from the filtered fields (to account for the presence of unresolved scales) which leads to the excellent results obtained in figure 13. Considering the degree of compressibility exhibited by the turbulence statistics for the test case considered in this section, it would appear that the feasibility of the proposed subgridscale models has been established. A more extensive parametric study of this subgrid-scale model is under investigation by Dahlburg et al. (1990).

\section{Conclusion}

New subgrid-scale models for compressible turbulence have been developed and tested against the results of direct numerical simulations of compressible isotropic turbulence. These compressible subgrid-scale models, which were based on the Favre-filtered equations of motion for an ideal gas, contain two dimensionless constants and reduce to the linear combination model of Bardina, Ferziger and Reynolds (1983) in the incompressible, isothermal limit. The subgrid-scale stress model constant was found to assume the value of $C_{R}=0.012$ which gives rise to correlations between the exact and modeled stresses that were above $70 \%$ on the tensor, vector and scalar levels - a correlation which compares favorably with those obtained in earlier work on the subgrid-scale modeling of incompressible turbulent flows. Another encouraging feature lies in the fact that these constants and their associated correlations were found to be comparatively insensitive to a mean Mach number in the range $0<M_{0}<0.6$.

The results of a coarse grid $32^{3}$ LES of compressible isotropic turbulence conducted with the subgrid-scale models derived in this paper were shown to be in excellent agreement with those obtained from a $96^{3}$ direct simulation. These results are extremely encouraging since, for the case considered, on average $25 \%$ of the turbulent kinetic energy was compressible. Furthermore, the ability for the LES to accurately capture the dilatational statistics of the flow was quite surprising.

Future research will be directed on several fronts. The large-eddy simulation of a compressible turbulent flow with mean temperature gradients could lead to refinements in the subgrid-scale heat flux model. Furthermore, the large-eddy simulation of compressible, homogeneous shear flow could yield new insights into the performance of these subgrid-scale models. Near-wall modifications will also be implemented that allow for the large-eddy simulation of compressible, wall-bounded turbulent flows. While further improvements are still possible, we believe that the essential foundation for the large-eddy simulation of compressible turbulent flows has been established in this study. With future research, compressible LES could have a profound impact on the analysis of supersonic and hypersonic flows of aerodynamic importance.

\section{References}

Arfken, G. 1970 Mathematical Methods for Physicists. Academic Press. 
Bardina, J., Ferziger, J. H. \& Reynolds, W. C. 1983 Improved Subgrid-Scale Models based on Large-Eddy Simulation of Homogeneous, Incompressible, Turbulent Flows. Stanford Report TF-19.

Batchelor, G. K. 1967 An Introduction to Fluid Dynamics. Cambridge University Press.

Biringen, S. \& Reynolds, W. C. 1981 Large-Eddy Simulation of the Shear-Free Turbulent Boundary Layer. J. Fluid Mech., 103, 53-63.

Canuto, C., Hussaini, M. Y., Quarteroni, A. \& Zang, T. A., 1987 Spectral Methods in Fluid Dynamics. Springer-Verlag.

Clark, R. A., Ferziger, J. H. \& Reynolds, W. C. 1979 Evaluation of Subgrid-Scale Models using an Accurately Simulated Turbulent Flow. J. Fluid Mech. 91, 1-16.

Comte-Bellot, G. \& Corrsin, S. 1971 Simple Eulerian Time Correlation of Full and NarrowBand Velocity Signals in Grid-Generated, Isotropic Turbulence. J. Fluid Mech. 48, 273-337.

Dahlburg, R.B., Zang, T.A. \& Dahlburg, J.P. 1990 Large-Eddy Simulations of Compressible, Isotropic Navier-Stokes Turbulence. Private Communication.

Deardorff, J. W. 1970 A Numerical Study of Three-Dimensional Turbulent Channel Flow at Large Reynolds Numbers. J. Fluid Mech. 41, 453-480.

Eidson, T.M. 1985 Numerical Simulation of Turbulent Rayleigh-Benard Problem using Numerical Subgrid Modeling. J. Fluid Mech. 158, 245-268.

Erlebacher, G., Hussaini, M.Y., Kreiss, H.O. \& Sarkar, S. 1990 The Analysis and Simulation of Compressible Turbulence. ICASE Report No. 90-15.

Feiereisen, W. J., Reynolds, W. C. \& Ferziger, J. H. 1981 Numerical Simulation of Compressible, Homogeneous, Turbulent Shear Flow. Report TF-13, Dept. Mech. Eng., Stanford University.

Frisch, U. \& Orszag, S. A. 1990 Turbulence: Challenges for Theory and Experiment. Physics Today 43, No. 1, 24-32.

Hinze, J. O. 1975 Turbulence. McGraw-Hill.

Hussaini, M. Y. \& Zang, T. A. 1987 Spectral Methods in Fluid Dynamics. Ann. Rev. Fluid Mech. 19, 339-368.

Hussaini, M.Y., Speziale, C.G. \& Zang, T.A. 1990 Comment on the Potential and Limitations of Direct and Large-Eddy Simulations, in Lecture Notes in Physics, 357, pp. 354-368, (ed. J.L. Lumley) Springer-Verlag.

Kerr, R. M. 1985 Higher-Order Derivative Correlations and the Alignment of Small-Scale Structures in Isotropic, Numerical Turbulence. J. Fluid Mech. 153, 31-58. 
Leonard, A., 1974 On the Energy Cascade in Large-Eddy Simulations of Turbulent Fluid Flows. Adv. Geophys. 18, 237-248.

Lumley, J. L. 1983 Turbulence Modeling. ASME J. Appl. Mech. 50, 1097-1103.

McMillan, O. J. 1980 Tests of New Subgrid-Scale Models in Strained Turbulence. AIAA paper AIAA-80-1339, in AIAA 13th Fluid and Plasma Dynamics Conference, Snowmass, Co.

McMillan, O. J. \& Ferziger, J. H. 1979 Direct Testing of Subgrid-Scale Models. AIAA J. 17, $1340-1346$.

Passot, T. \& Pouquet, A. 1987 Numerical Simulation of Compressible Homogeneous Flows in the Turbulent Regime. J. Fluid Mech. 181, 441-466.

Piomelli, U., Ferziger, J. H. \& Moin, P. 1987 Models for Large-Eddy Simulations of Turbulent Channel Flows including Transpiration. Stanford University Technical Report TF-32.

Piomelli, U., Zang, T. A., Speziale, C. G. \& Hussaini, M. Y. 1990 On the Large-Eddy Simulation of Transitional Wall-Bounded Flows. Phys. Fluids A, 2, 257-265.

Reynolds, W. C. 1976 Computation of Turbulent Flows. Ann. Rev. Fluid Mech. 8, 183-208.

Rogallo, R. S. \& Moin, P. 1984 Numerical Simulation of Turbulent Flows. Ann. Rev. Fluid Mech. 16, 99-137.

Sarkar, S.; Erlebacher, G., Hussaini, M.Y. \& Kreiss, H.O. 1989 The Analysis and Modeling of Dilatational Terms in Compressible Turbulence. ICASE Report No. 89-79.

Schumann, U. 1975 Subgrid Scale Model for Finite Difference Simulations of Turbulent Flows in Plane Channels and Annuli. J. Comput. Phys. 18, 376-404.

Speziale, C. G. 1985 Galilean Invariance of Subgrid-Scale Stress Models in the Large-Eddy Simulation of Turbulence. J. Fluid Mech. 156, 52-62.

Speziale, C.G., Erlebacher, G., Zang, T.A. \& Hussaini, M.Y. 1988 The Subgrid-Scale Modeling of Compressible Turbulence. Phys. Fluids 31, 940-942.

Tennekes, H. \& Lumley, J. L. 1972 A First Course in Turbulence. MIT press.

Voke, P. R. \& Collins, M. W. 1983 Large-Eddy Simulation: Retrospect and Prospect. PhysicoChemical Hydrodynamics 4, No. 2, 119-161.

Yoshizawa, A. 1986 Statistical Theory for Compressible Turbulent Shear Flows, with the Application to Subgrid Modeling. Phys. Fluids A 29, 2152-2164. 


\section{Appendix}

\section{Initial energy spectrum}

Both the incompressible and compressible direct simulations impose a specified energy spectrum on the initial random velocity distribution. For comparative purposes, the data was obtained from tabular data found in Comte-Bellot and Corrsin (1971). They tabulate the function $E_{11}(k)$ which is related to the energy spectrum $E(k)$ by

$$
E(k)=\frac{1}{2} k^{3} \frac{d}{d k}\left[\frac{1}{k} \frac{d E_{11}}{d k}\right] .
$$

Unfortunately the data is noisy, so a least squares fit is performed on $\log E_{11}$, expressed as a fourth order polynomial in $\log k$. The final form obtained for $E_{11}$ is

$$
\begin{aligned}
\log \left(E_{11}\right)= & 2.64359-0.72602(\log k)-0.32585(\log k)^{2} \\
& +0.03525(\log k)^{3}-0.02344(\log k)^{4} .
\end{aligned}
$$

\section{Calculation of model constants}

Before correlating the total exact subgrid-scale stress against its model, the model constants must be determined. There are many ways of accomplishing this among which two are retained. The total modeled stress is written as a linear combination of modeled terms $C_{i} \tau_{i}^{m}$

$$
\tau^{m}=\sum_{i=1}^{n} C_{i} \tau_{i}^{m}
$$

while the exact total stress is simply

$$
\tau^{e}=\sum_{i=1}^{n} \tau_{i}^{e}
$$

The unknown constants to be determined are the $C_{i}$. The first approach adopted is to calculate the root mean square of the pairs $C_{i} \tau_{i}^{m}$ and $\tau_{i}^{e}$ and equate them for each value of $i$. The constants thus take the values

$$
C_{i}=\frac{\sigma_{\tau_{i}^{e}}}{\sigma_{\tau_{i}^{m}}}
$$

This method is referred to by RMS in the text.

A least square method applied to the total stress as a whole is an alternative approach. In this case, the norm

$$
\left\|\tau^{m}-\tau^{e}\right\|^{2}=\left\|\sum_{i=1}^{n}\left(\tau_{i}^{e}-C_{i} \tau_{i}^{e}\right)\right\|^{2}
$$

is minimized with respect to the coefficients $C_{\boldsymbol{i}}$. This gives rise to a linear system in the coefficients which can be solved by direct methods if the number of constants is not too large. For the subgrid-scale model considered in the text, $n=3$. 


\section{Definitions}

For reference purposes, several statistical definitions are provided here. All variables are defined on a three-dimensional grid and are subscripted by a single index $i$ for convenience. The average of a function $\mathcal{F}_{i}$ is

$$
\langle\mathcal{F}\rangle=\frac{1}{N} \sum_{i=1}^{N} \mathcal{F}_{i} .
$$

As a function of the average, the rms of $\mathcal{F}$ is

$$
\mathcal{F}_{\text {rms }}=\sqrt{\left\langle(\mathcal{F}-\langle\mathcal{F}\rangle)^{2}\right\rangle}
$$

Correlation coefficients are fundamental in evaluating subgrid-scale models. The correlation coefficient between two functions $\mathcal{F}$ and $\mathcal{G}$ is

$$
C(\mathcal{F}, \mathcal{G})=\frac{\langle\mathcal{F G}\rangle}{\mathcal{F}_{\text {rms }} \mathcal{G}_{\text {rms }}}
$$




\section{List of Figures}

Fig. 1 Energy spectra at $t=0.0586$ for both aliased and de-aliased calculations for $64^{3}, 96^{3}$, and $128^{3}$ grids.

Fig. 2 Three-dimensional Mach 1.0 contours of the DNS at $t=0.0586$. The direct simulation was performed on a $96^{3}$ grid.

Fig. 3 Time history of one third the trace of Sk for $M_{0}$ of $0.0,0.1,0.4$ and 0.6 . Direct simulation was performed on a $96^{3}$ grid. CBC initial conditions.

Fig. 4 Time history of one third the trace of $\lambda_{i j}$ for $M_{0}$ of $0.0,0.1,0.4$ and 0.6 . Direct simulation was performed on a $96^{3}$ grid. CBC initial conditions.

Fig. 5 Time history of kinetic energy for $M_{0}$ of $0.0,0.1,0.4$ and 0.6 . Direct simulation was performed on a $96^{3}$ grid. CBC initial conditions.

Fig. 6 Time history of one third the trace of Fl for $M_{0}$ of $0.0,0.1,0.4$ and 0.6 . Direct simulation was performed on a $96^{3}$ grid. CBC initial conditions.

Fig. 7 Time history of $R_{\lambda}$ for $M_{0}$ of $0.0,0.1,0.4$ and 0.6 . Direct simulation was performed on a $96^{3}$ grid. CBC initial conditions.

Fig. 8 Energy and dissipation spectra of $96^{3}$ DNS at $t=0,0.2,0.4,0.8,1.6 . k_{0}=10, \chi_{0}=0.0$, $\left(M_{t}\right)_{0}=0.1,\left(R_{\lambda}\right)_{0}=30.0, R e=250$.

(a) Dissipation, (b) Compressible energy, (c) Solenoidal energy.

Fig. 9 Energy and dissipation spectra of $96^{3} \mathrm{DNS}$ at $t=0,0.2,0.4,0.8,1.6 . k_{0}=10, \chi_{0}=0.2$, $\left(M_{t}\right)_{0}=0.1,\left(R_{\lambda}\right)_{0}=26.4, R e=250$.

(a) Dissipation, (b) Compressible energy, (c) Solenoidal energy.

Fig. 10 Turbulence statistics for $96^{3}$ DNS at $t=0.8$. Parameters are $k_{0}=10, \chi_{0}=0.0$ and $\chi_{0}=0.2,\left(M_{t}\right)_{0}=0.1,\left(\rho_{r m s}\right)_{0}=0,\left(T_{r m s}\right)_{0}=0.0626, R e=250$.

(a) $E_{T O T}=E^{C}+E^{I}$, (b) trace of skewness tensor, (c) trace of flatness tensor, (d) trace of Taylor length tensor, (e) integral length scale, (f) microscale Reynolds number.

Fig. 11 Volumetric representation of $\omega^{2}$. Parameters are identical to those of Fig. 10.

Fig. 12 Volumetric representation of $(\nabla \cdot v)^{2}$. Parameters are identical to those of Fig. 10.

Fig.13 Comparison between LES results (-, - - , ---) and results from a $96^{3}$ DNS $(\triangle, \square, \diamond)$ injected onto the $32^{3}$ LES grid. Filter width $\Delta_{c}=\Delta_{f}=2$.

Fig.14 Comparison between LES results (-, -- - - - ) and results from a $96^{3}$ DNS $(\triangle, \square, \diamond)$ injected onto the $32^{3}$ LES grid. Filter width $\Delta_{c}=\Delta_{f}=0$. 

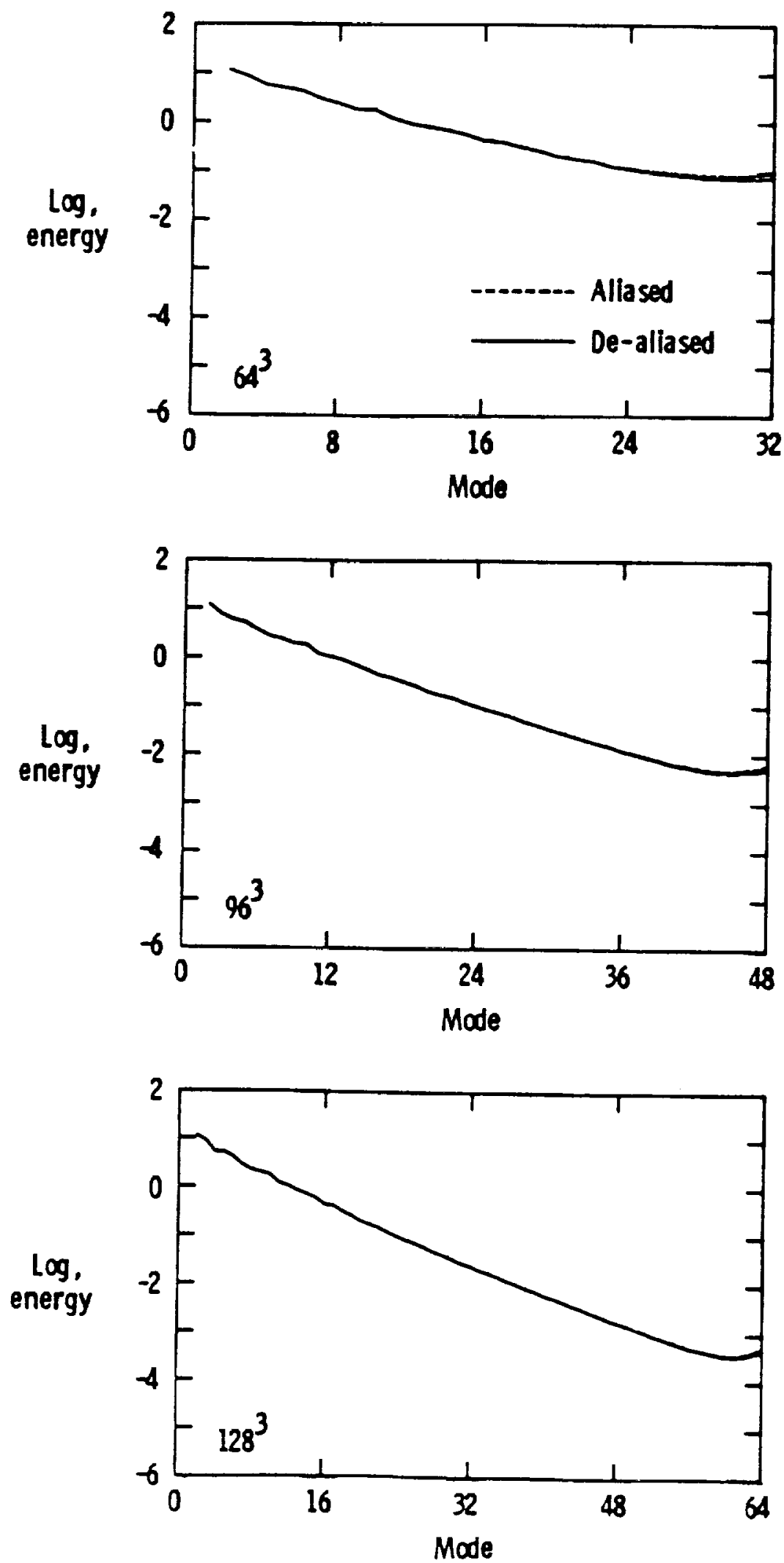

Fig. 1 Energy spectra at $t=0.0586$ for both aliased and dealiased calculations for $64^{3}, 96^{3}$, and $128^{3}$ grids. 


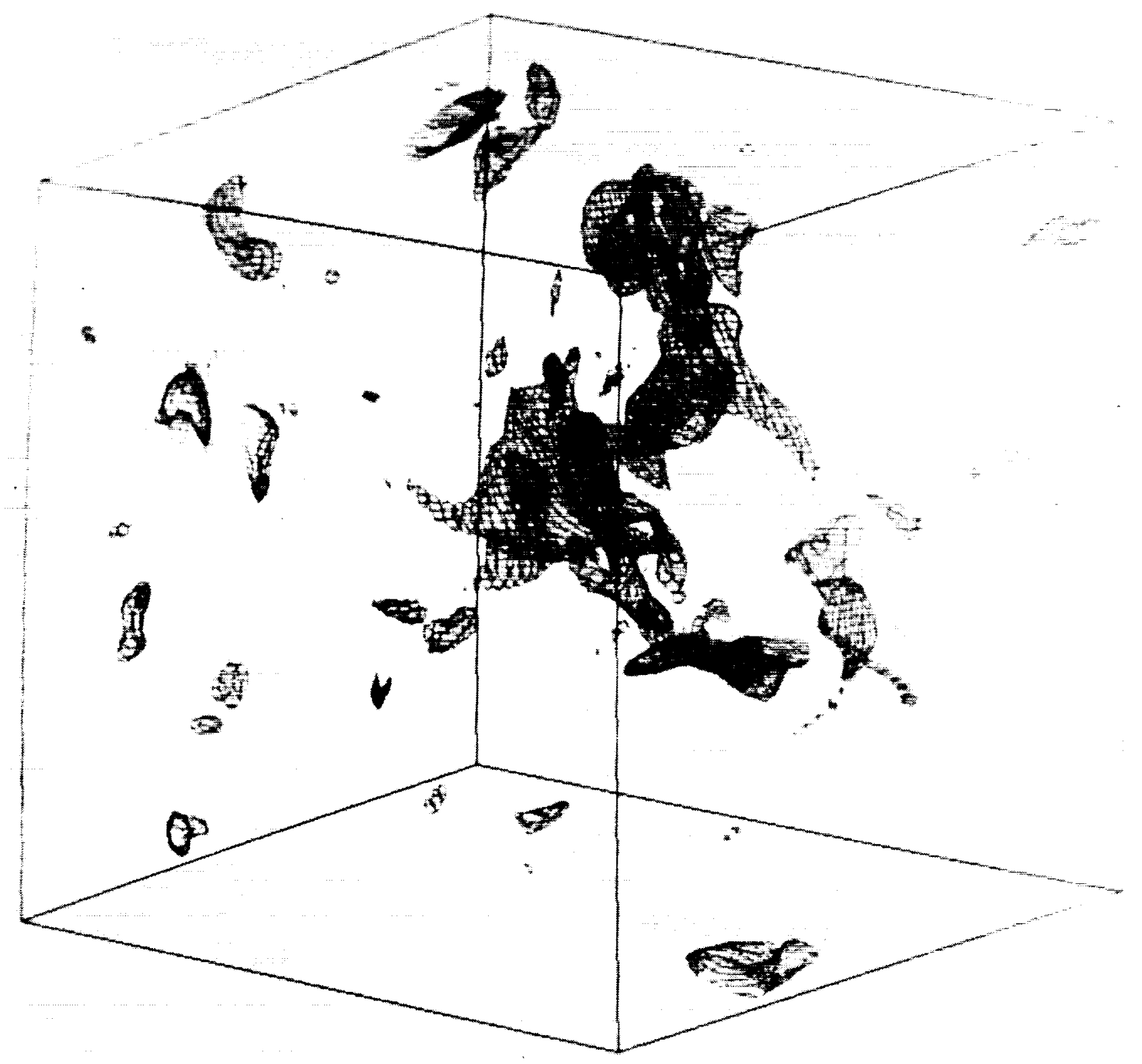

Fig. 2 Three-dimensional Mach 1.0 contours of the DNS at $t=0.0586$. The direct simulation was performed on a $96^{3}$ grid. 


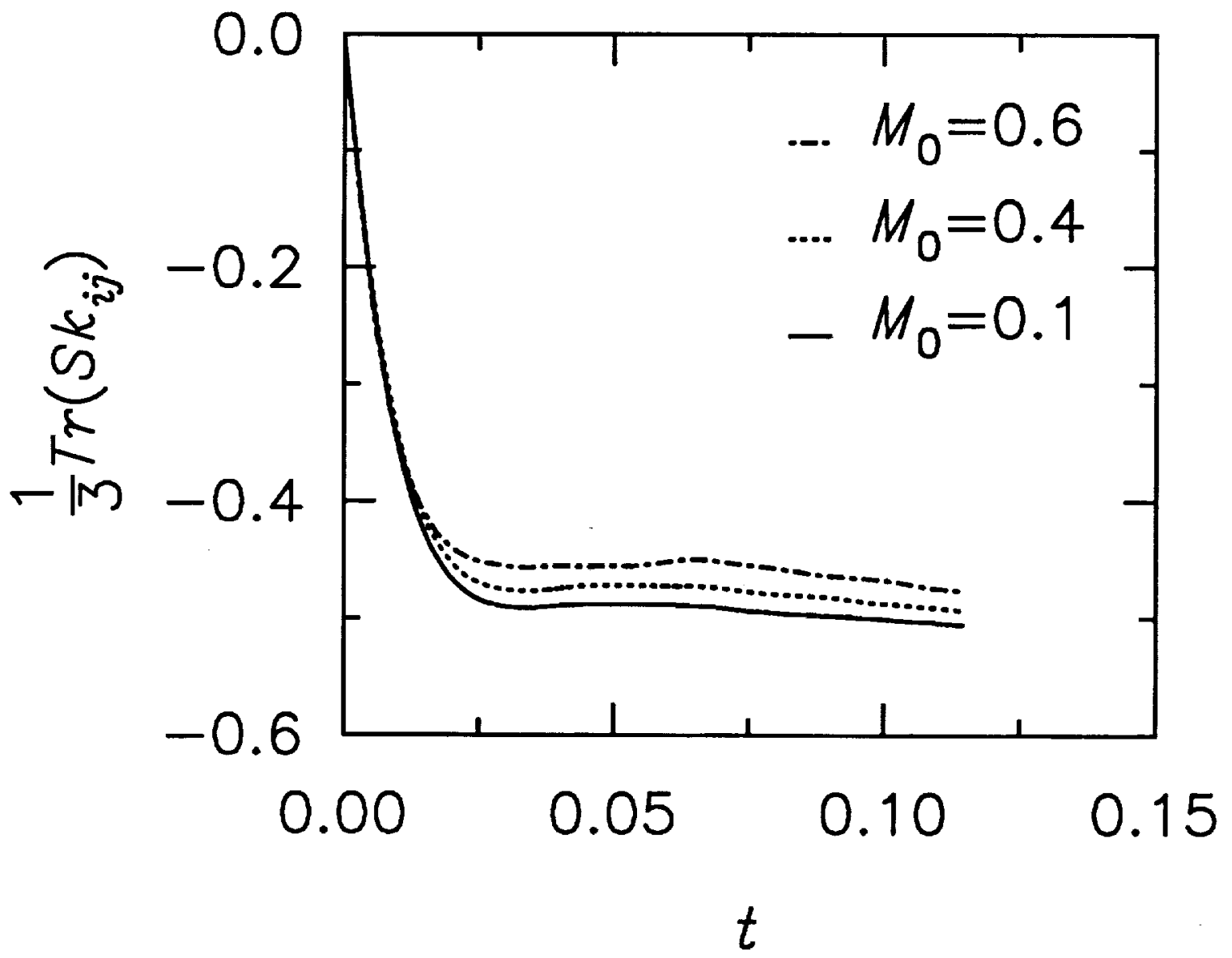

Fig. 3 Time history of one third the trace of Sk for $M_{0}$ of $0.0,0.1,0.4$ and 0.6 . Direct simulation was performed on a $96^{3}$ grid. CBC initial conditions. 


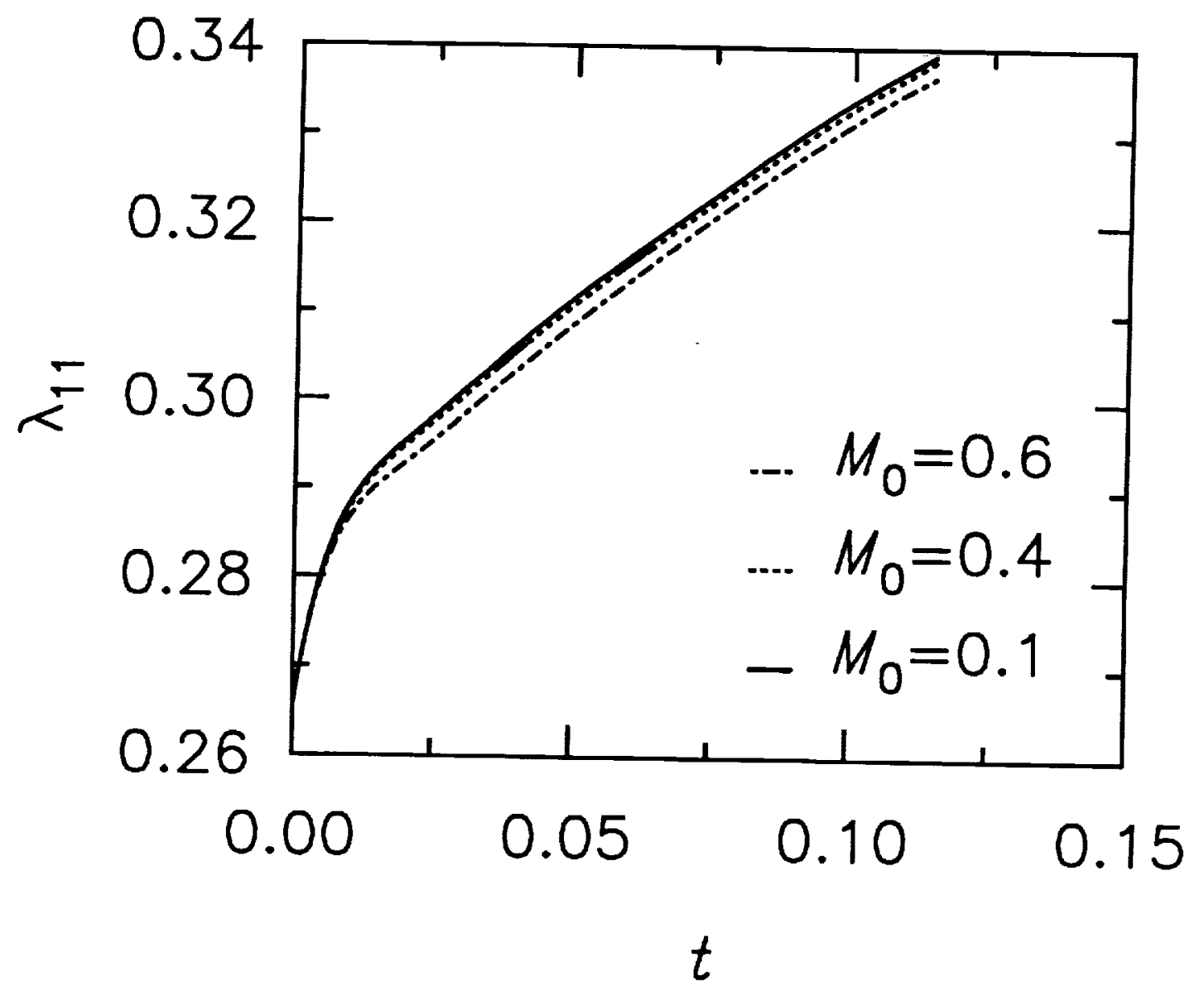

Fig. 4 Time history of one third the trace of $\lambda_{i j}$ for $M_{0}$ of $0.0,0.1,0.4$ and 0.6 . Direct simulation was performed on a $96^{3}$ grid. CBC initial conditions. 


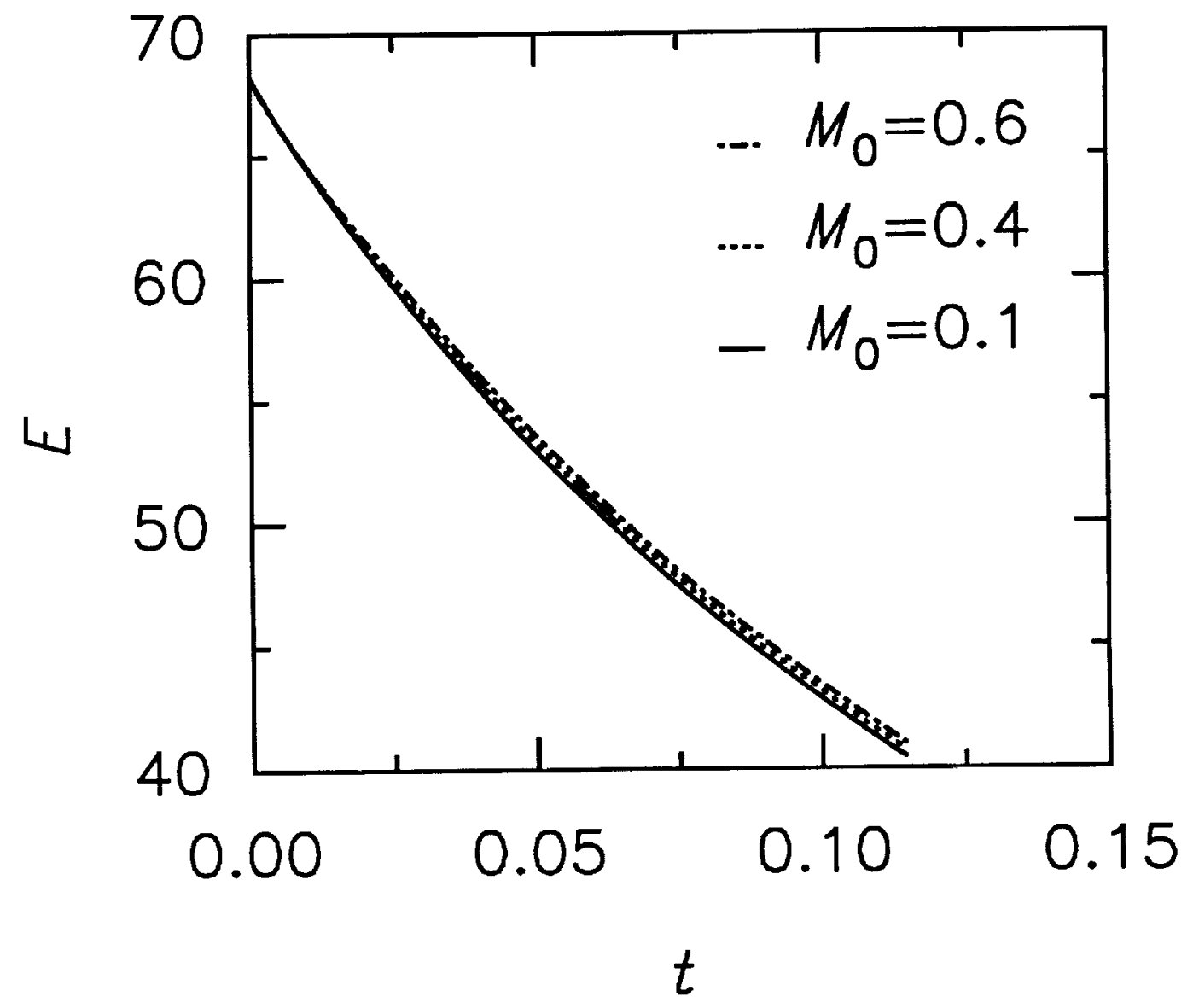

Fig. 5 Time history of kinetic energy for $M_{0}$ of $0.0,0.1,0.4$ and 0.6 . Direct simulation was performed on a $96^{3}$ grid. CBC initial conditions. 


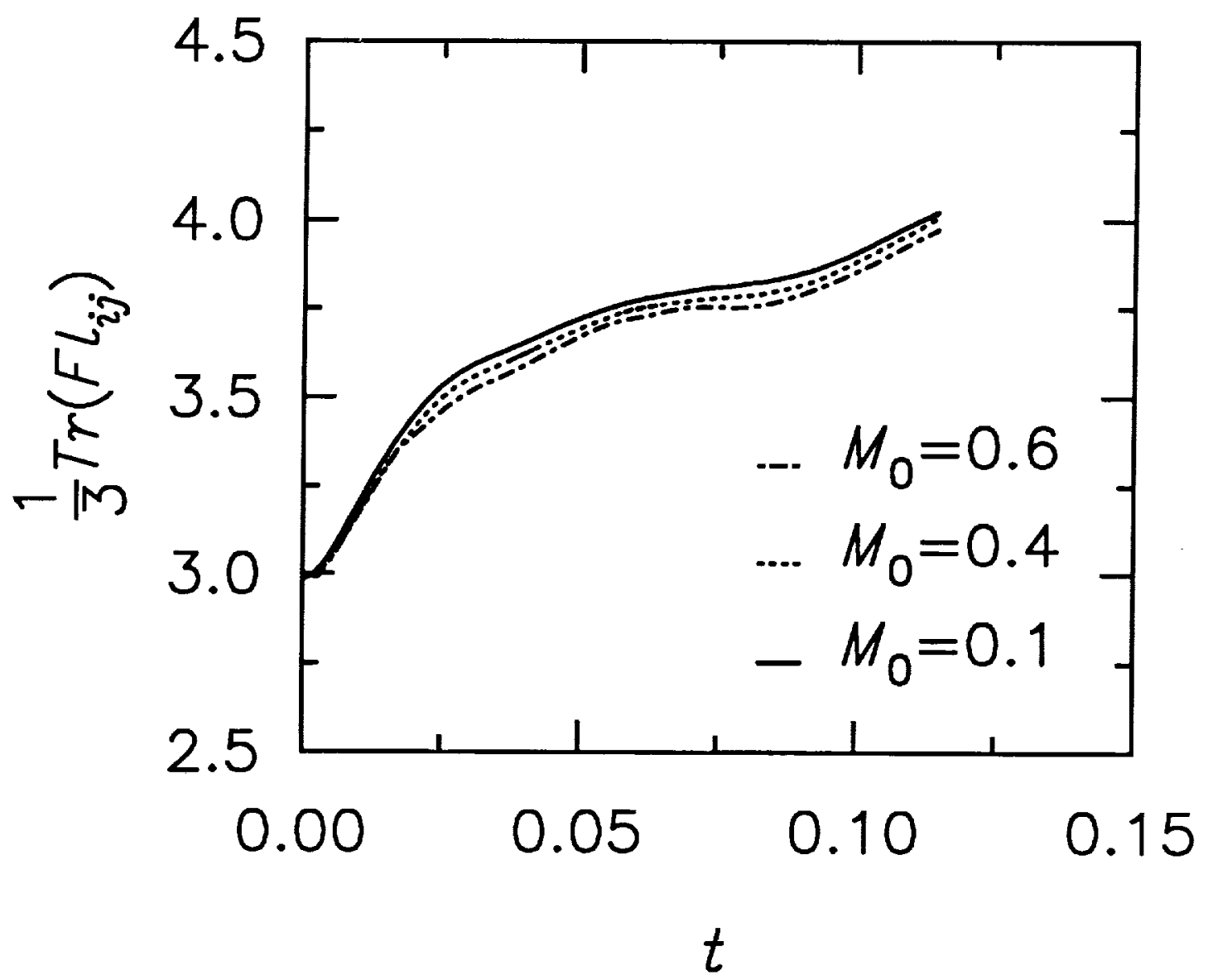

Fig. 6 Time history of one third the trace of Fl for $M_{0}$ of $0.0,0.1,0.4$ and 0.6. Direct simulation was performed on a $96^{3}$ grid. CBC initial conditions. 


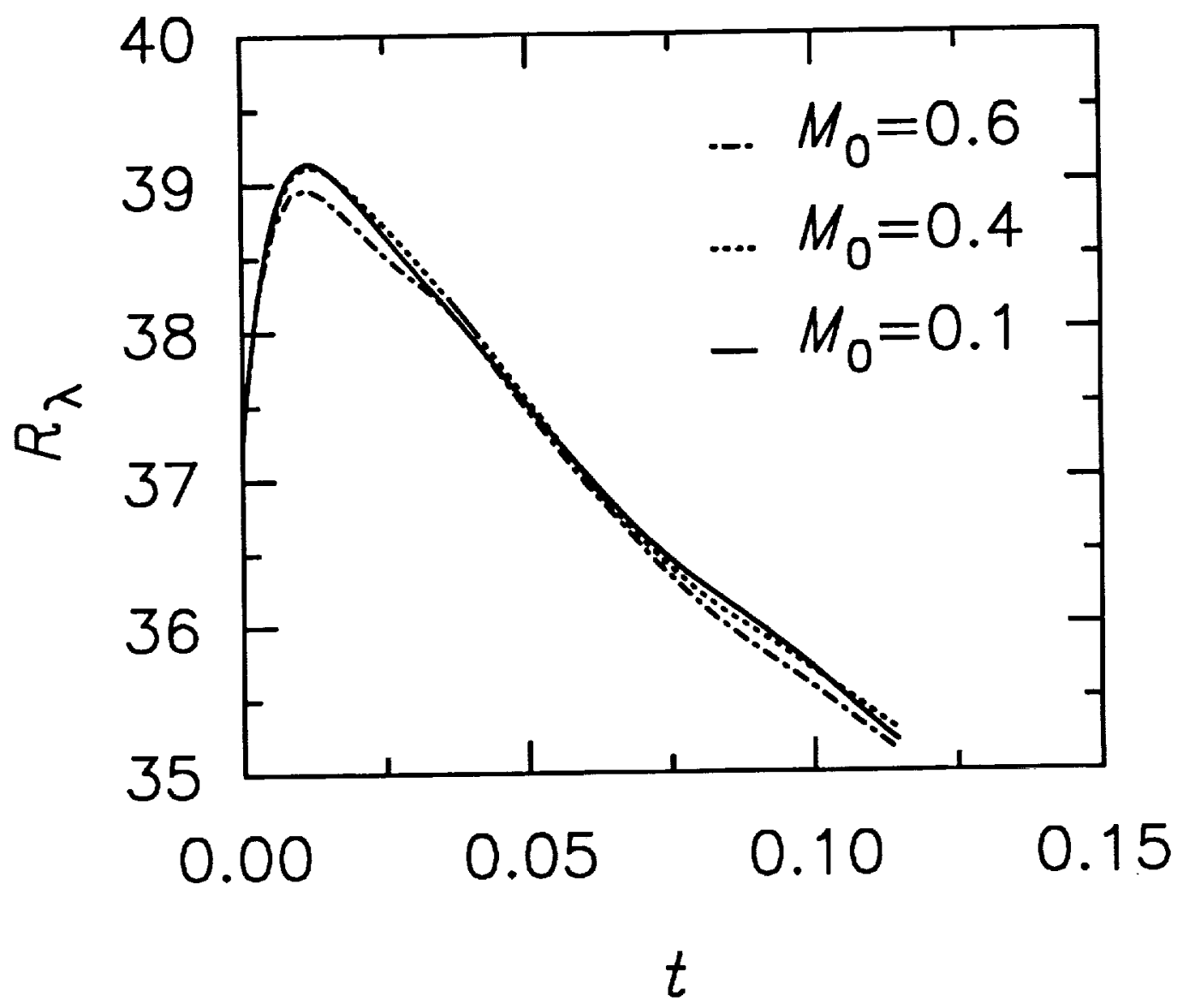

Fig. 7 Time history of $R_{\lambda}$ for $M_{0}$ of $0.0,0.1,0.4$ and 0.6 . Direct simulation was performed on a $96^{3}$ grid. CBC initial conditions. 
(a)

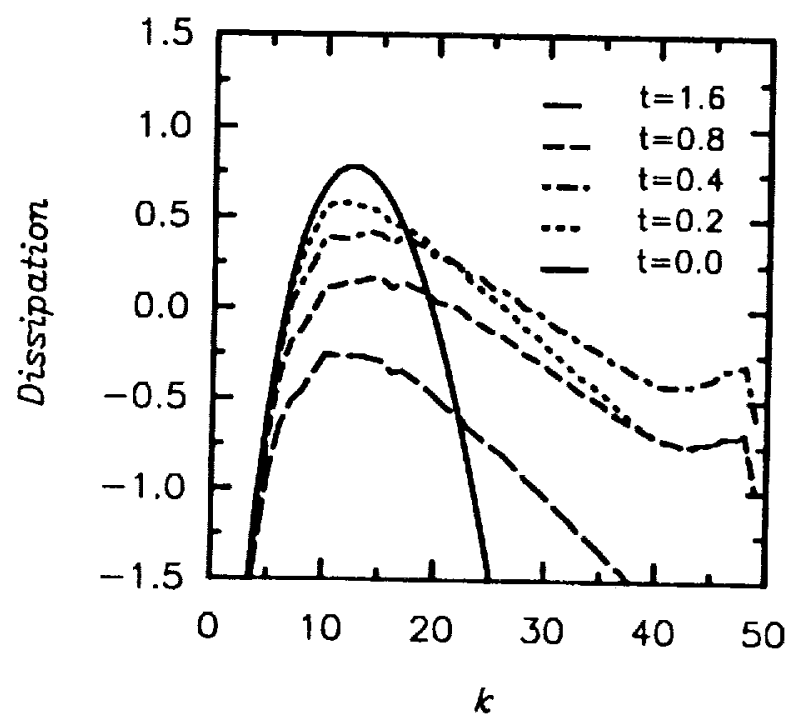

(b)

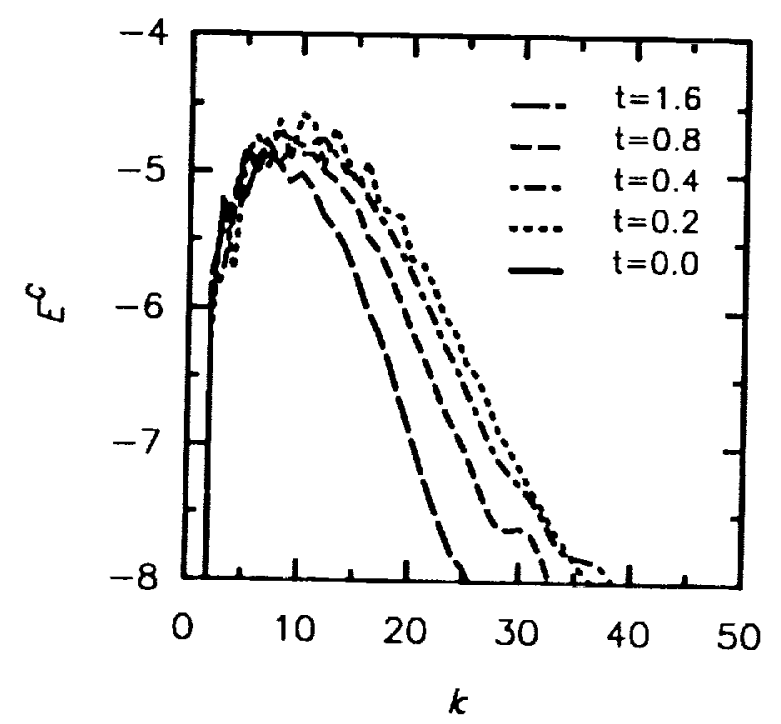

(c)

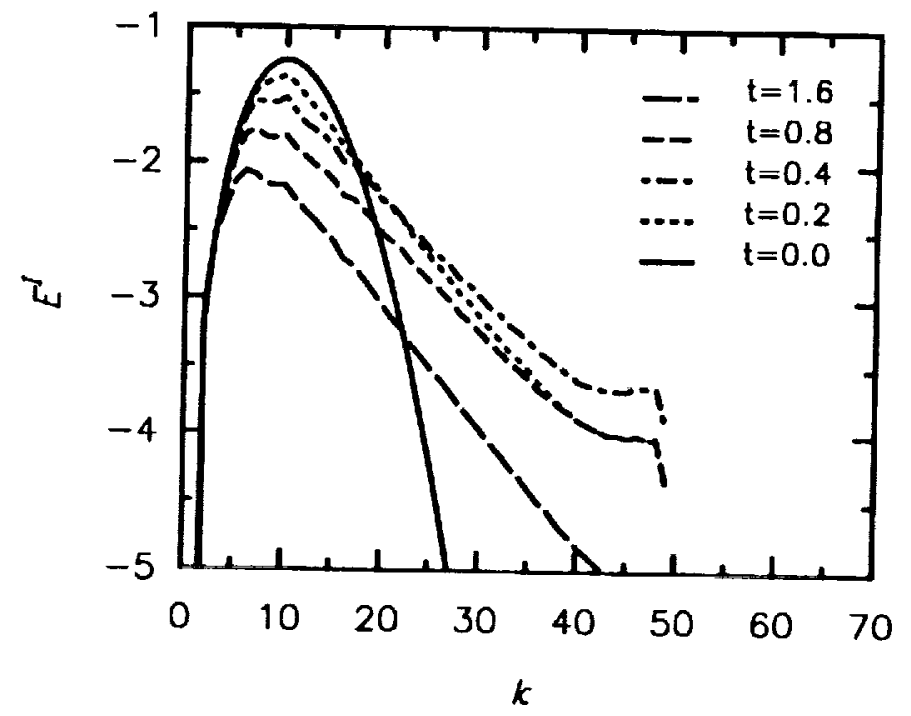

Fig. 8 Energy and dissipation spectra of $96^{3}$ DNS at $t=0,0.2,0.4,0.8,1.6 . k_{0}=10, \chi_{0}=0.0$, $\left(M_{t}\right)_{0}=0.1,\left(R_{\lambda}\right)_{0}=30.0, R e=250$.

(a) Dissipation, (b) Compressible energy, (c) Solenoidal energy. 
(a)

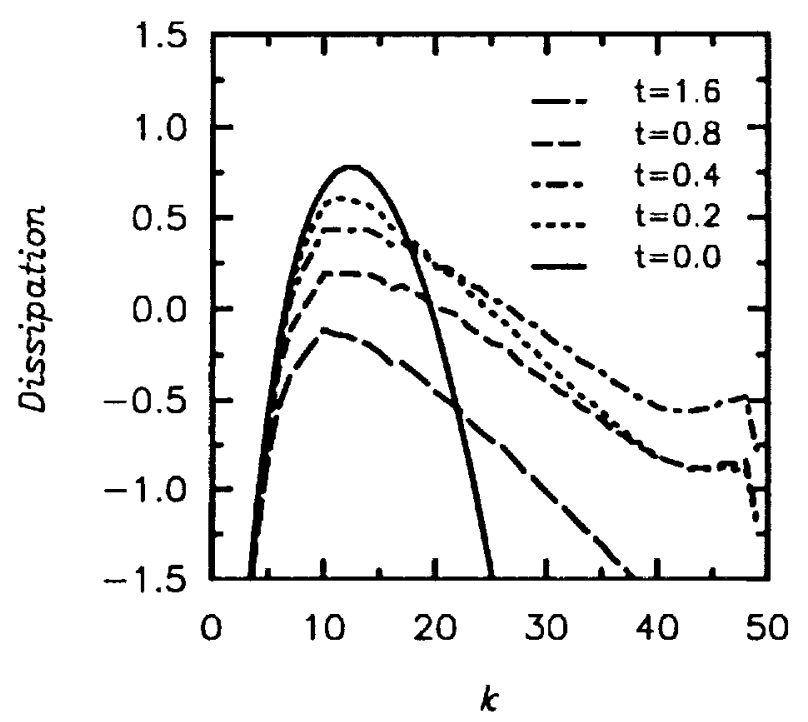

(b)

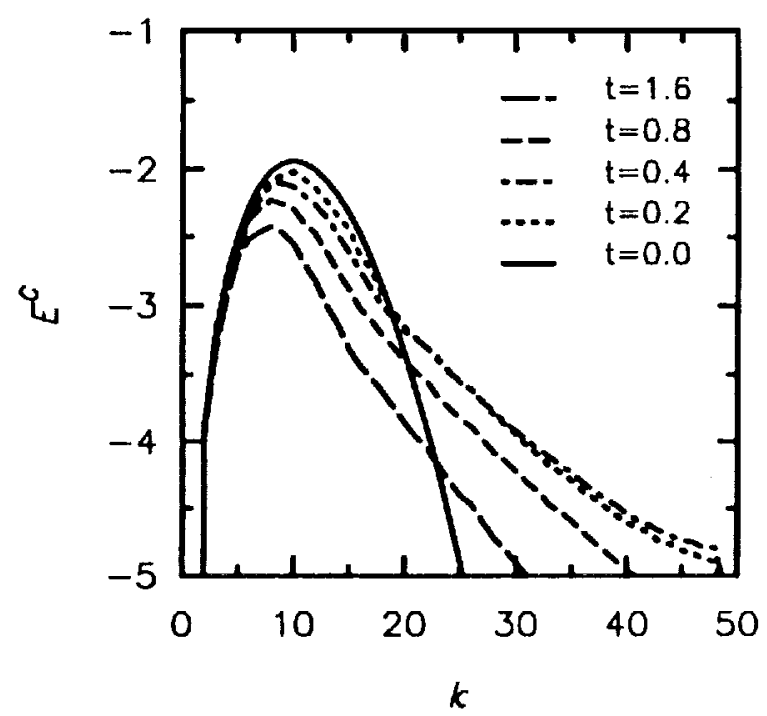

(c)

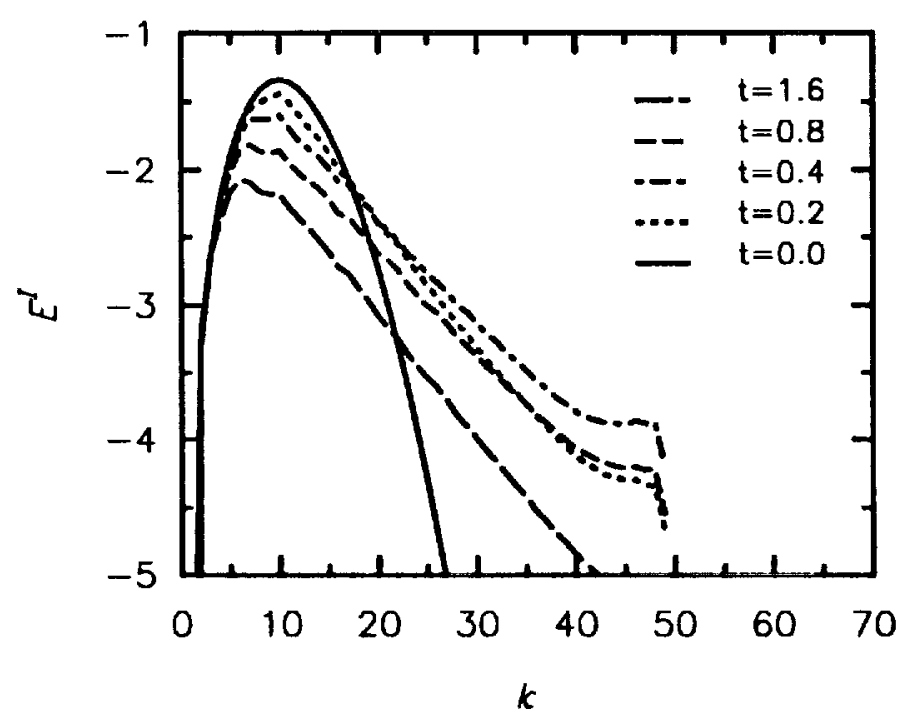

Fig. 9 Energy and dissipation spectra of $96^{3}$ DNS at $t=0,0.2,0.4,0.8,1.6 . k_{0}=10, \chi_{0}=0.2$, $\left(M_{t}\right)_{0}=0.1,\left(R_{\lambda}\right)_{0}=26.4, R e=250$.

(a) Dissipation, (b) Compressible energy, (c) Solenoidal energy. 
(a)

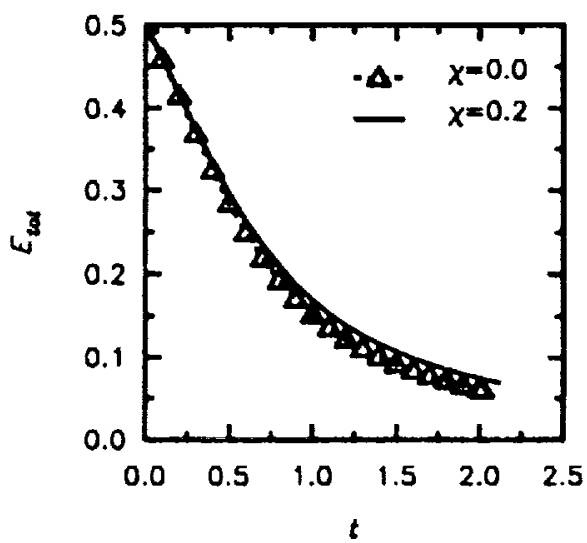

(c)

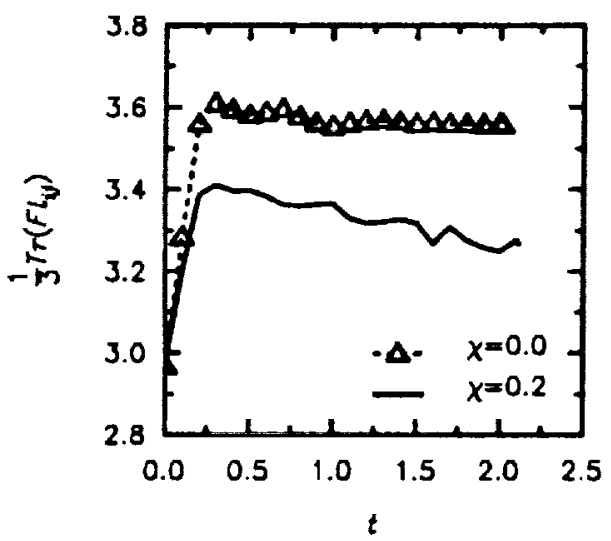

(e)

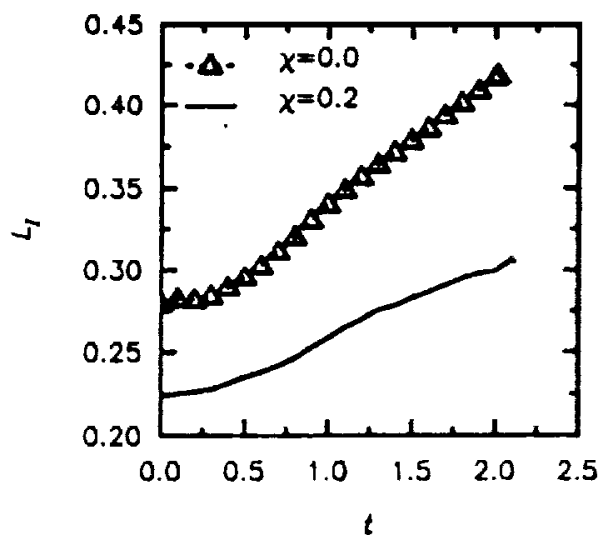

(b)

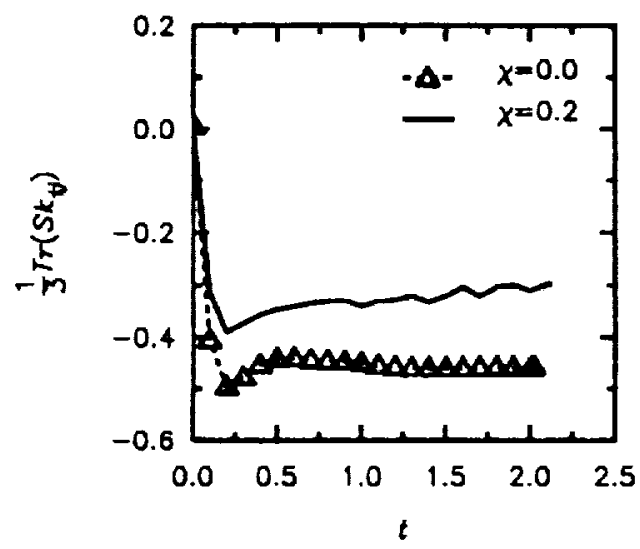

(d)

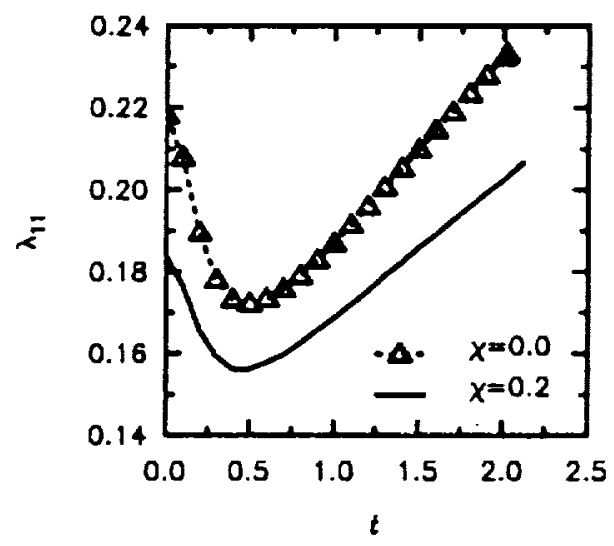

(f)

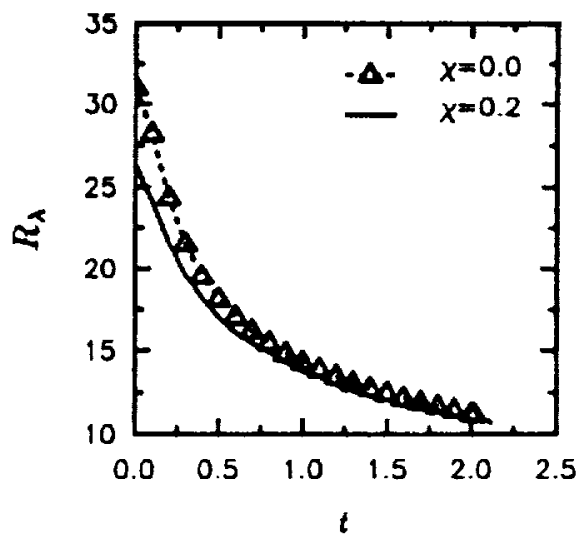

Fig. 10 Turbulence statistics for $96^{3}$ DNS at $t=0.8$. Parameters are $k_{0}=10, \chi_{0}=0.0$ and $\chi_{0}=0.2,\left(M_{t}\right)_{0}=0.1,\left(\rho_{\text {rms }}\right)_{0}=0,\left(T_{r m s}\right)_{0}=0.0626, R e=250$.

(a) $E_{T O T}=E^{C}+E^{I}$, (b) trace of skewness tensor, (c) trace of flatness tensor, (d) trace of Taylor length tensor, (e) integral length scale, (f) microscale Reynolds number. 


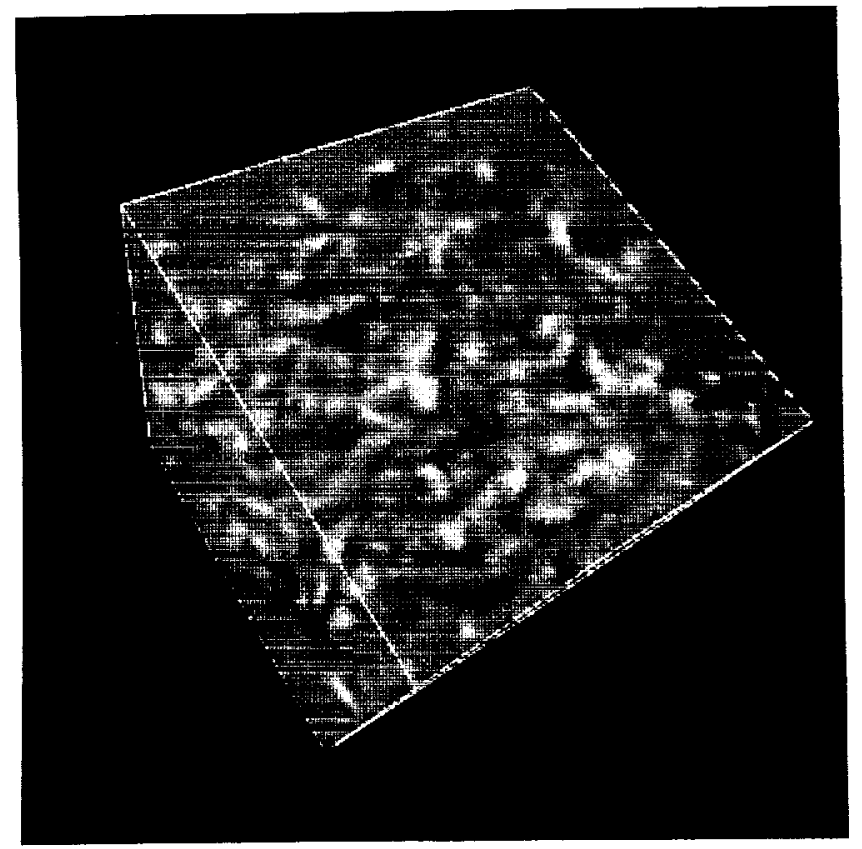

Fig. 11 Volumetric representation of $\boldsymbol{\omega}^{2}$. Parameters are identical to those of Fig. 10.

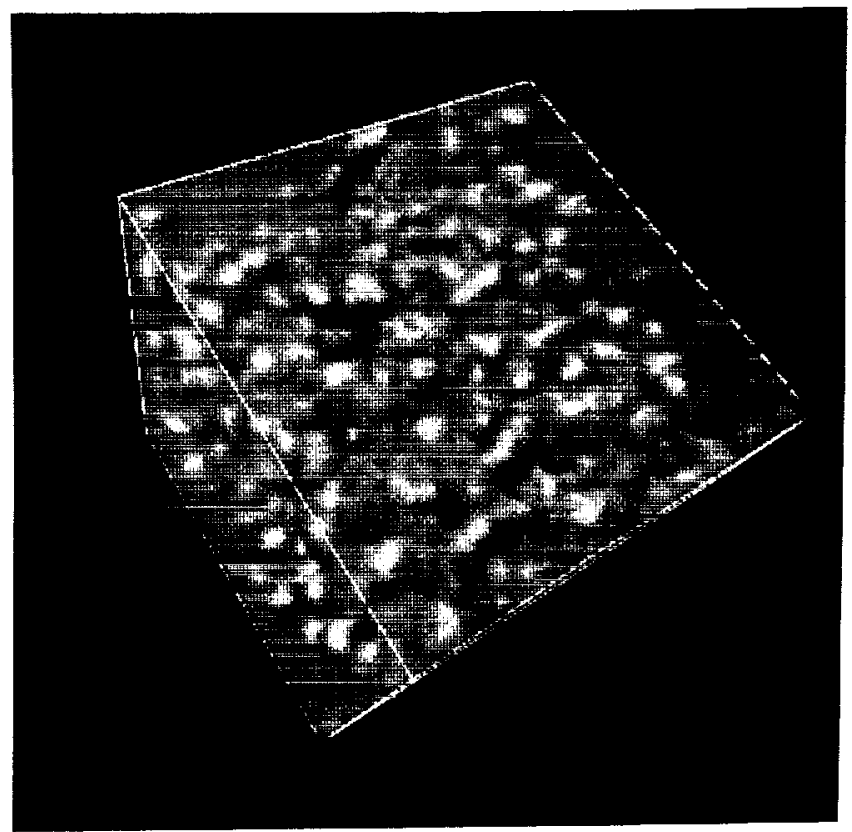

Fig. 12 Volumetric representation of $(\nabla \cdot \mathbf{v})^{2}$. Parameters are identical to those of Fig. 10. 
(a)

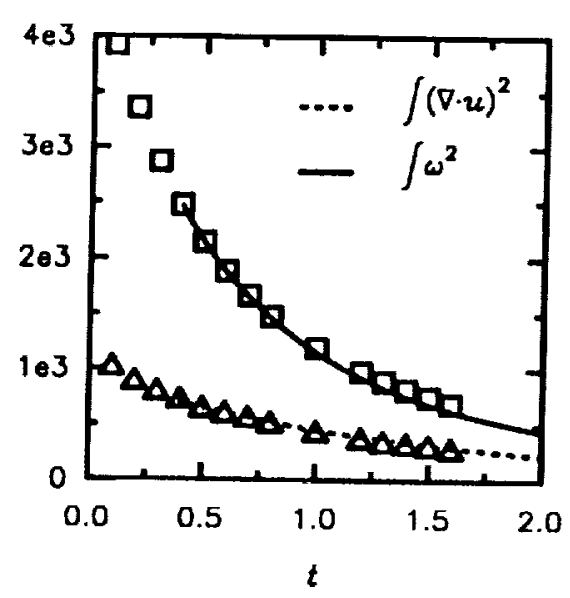

(c)

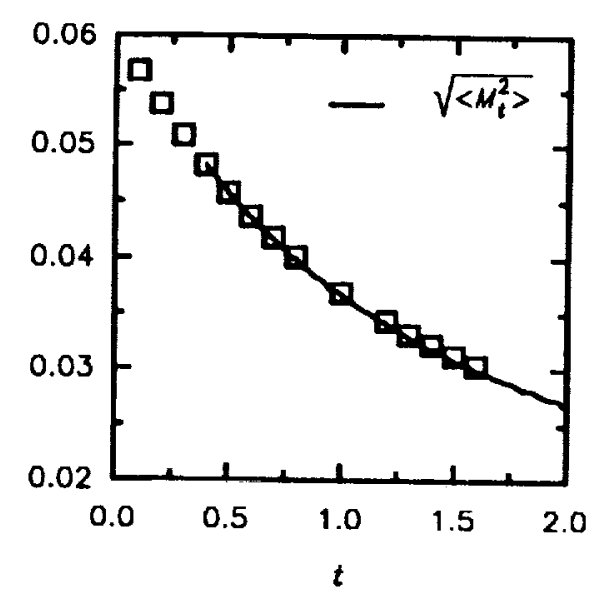

(e)

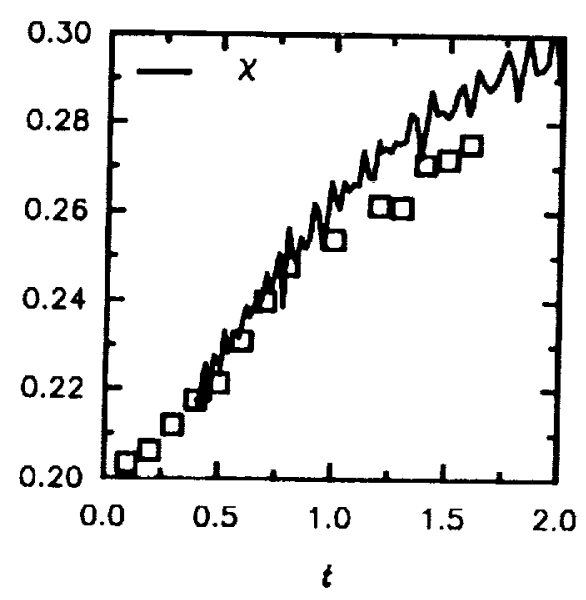

(b)

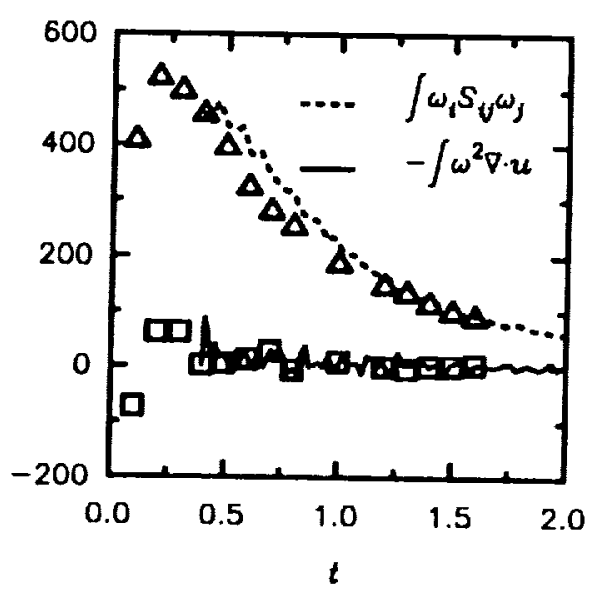

(d)

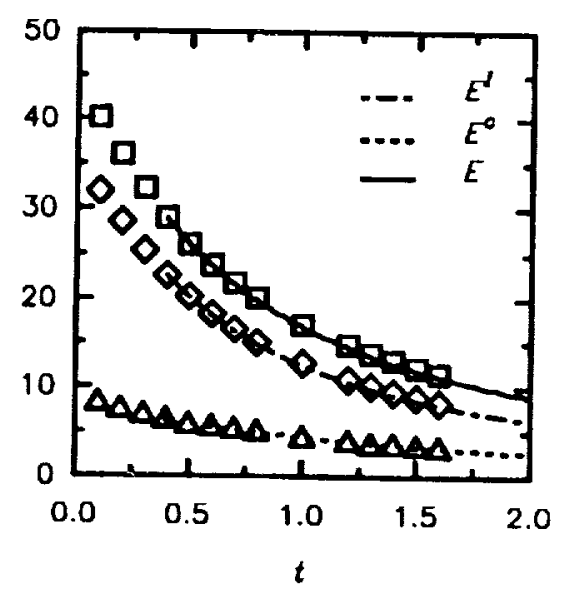

(f)

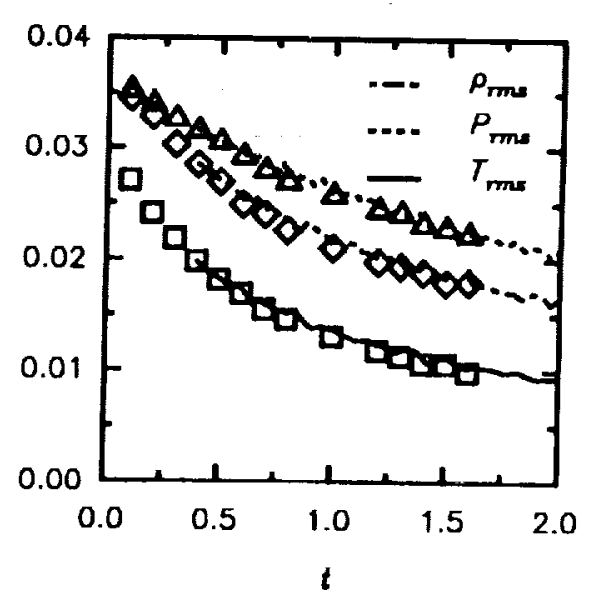

Fig.13 Comparison between LES results (-, ---, ---) and results from a $96^{3}$ DNS $(\triangle, \square, \diamond)$ injected onto the $32^{3}$ LES grid. Filter width $\Delta_{c}=\Delta_{f}=2$. 
(a)

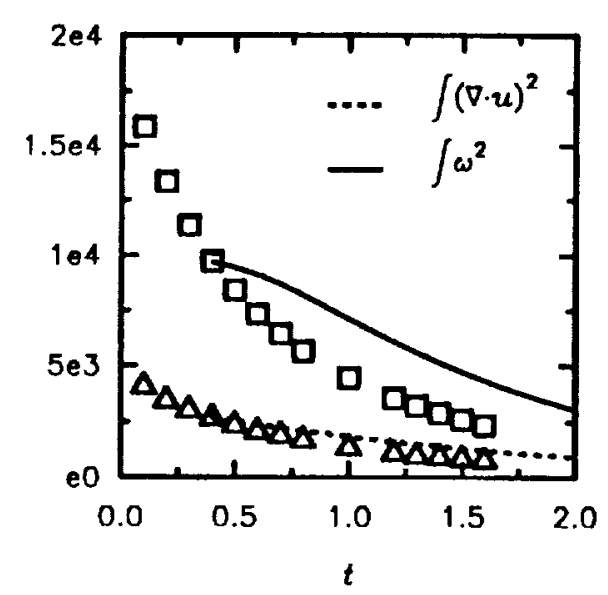

(c)

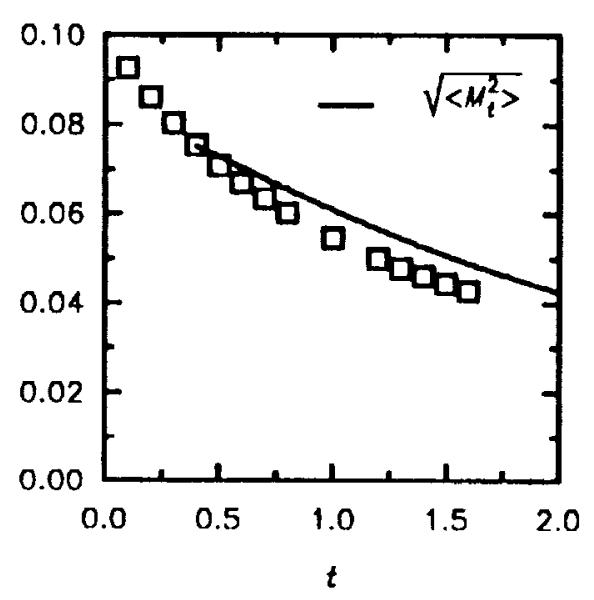

(e)

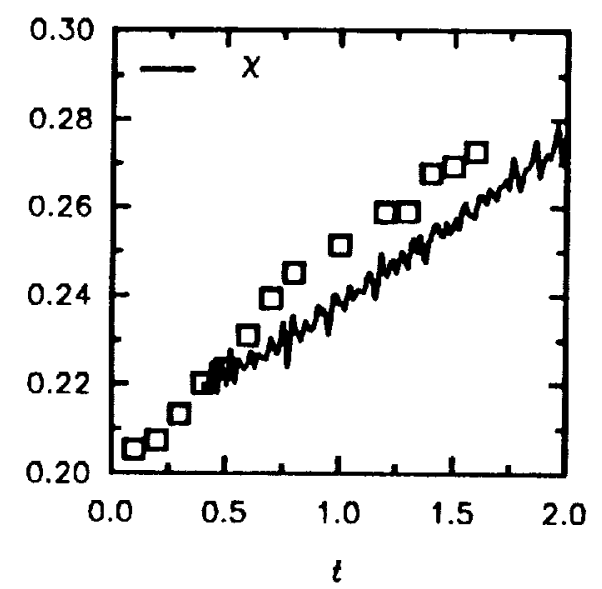

(b)

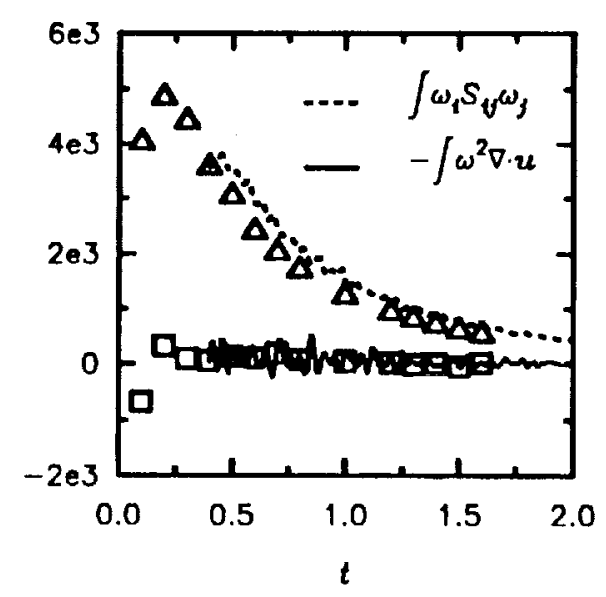

(d)

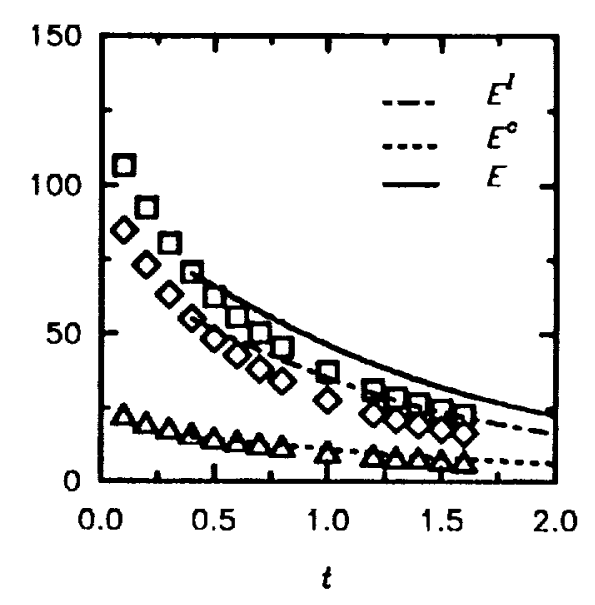

(f)

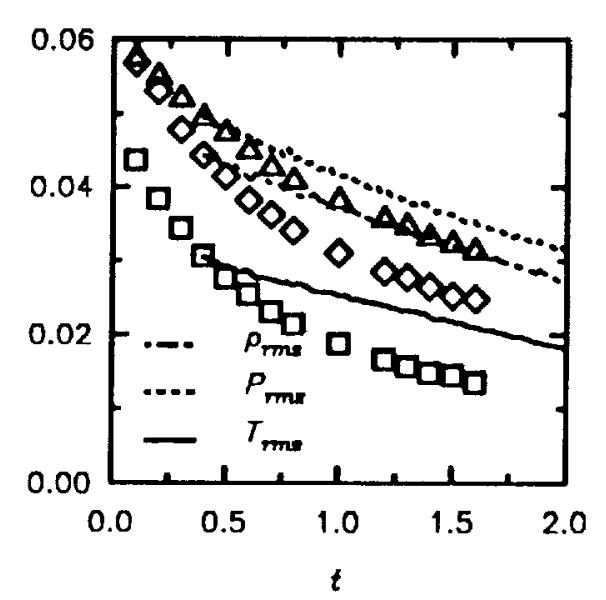

Fig.14 Comparison between LES results (- - - - - - ) and results from a $96^{3}$ DNS $(\triangle, \square, \diamond)$ injected onto the $32^{3}$ LES grid. Filter width $\Delta_{c}=\Delta_{f}=0$. 



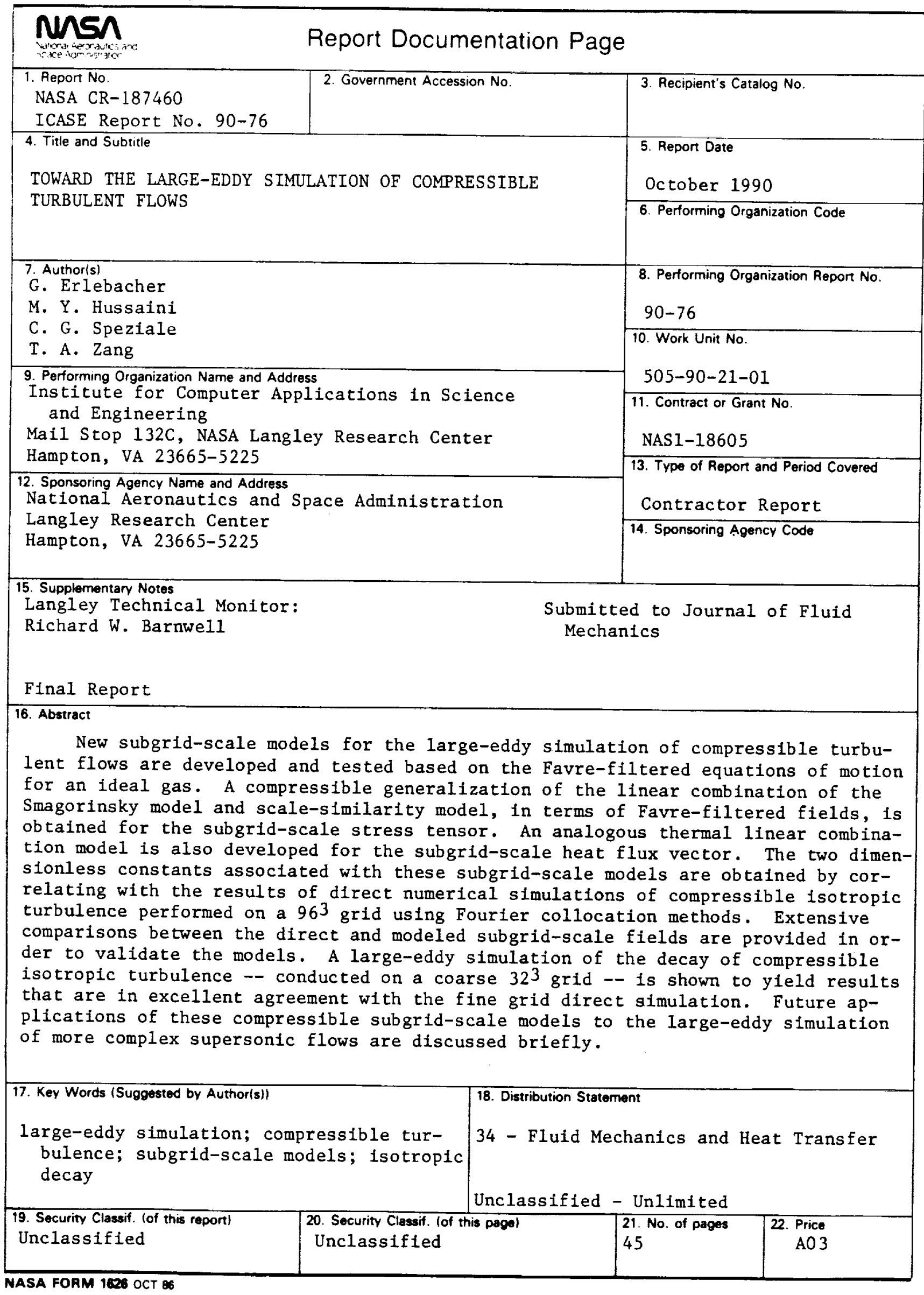


